\title{
PAPER
}

\section{Camera-based CW Diffuse Optical Tomography for obtaining 3D absorption maps by means of digital tomosynthesis}

To cite this article: N A Carbone et al 2020 Biomed. Phys. Eng. Express 6065034

View the article online for updates and enhancements.

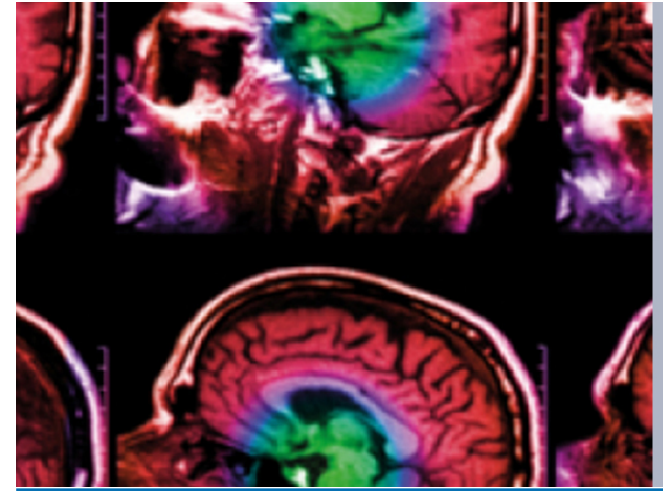

\section{IPEM IOP}

Series in Physics and Engineering in Medicine and Biology

Your publishing choice in medical physics,

biomedical engineering and related subjects.

Start exploring the collection-download the first chapter of every title for free.

This content was downloaded from IP address 131.221 .0 .8 on 14/12/2020 at 12:33 


\title{
Biomedical Physics \& Engineering Express
}

\section{PAPER}

CrossMark

\section{Camera-based CW Diffuse Optical Tomography for obtaining 3D absorption maps by means of digital tomosynthesis}

7 July 2020

REVISED

16 September 2020

ACCEPTED FOR PUBLICATION

30 October 2020

PUBLISHED

10 November 2020

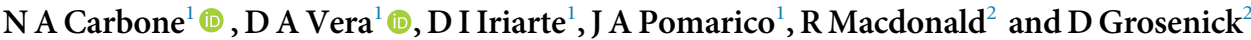 \\ 1 CIFICEN (UNCPBA-CONICET-CICPBA), Pinto 399, B7000GHG Tandil, Argentina \\ 2 Physikalisch-Technische Bundesanstalt(PTB), Abbestraße 2-12, 10587 Berlin, Germany \\ E-mail:ncarbone@exa.unicen.edu.ar
}

Keywords: multiple scattering, tissue optics, CW, tomosynthesis, NIR

\begin{abstract}
We present a novel method for obtaining a 3D absorption map of a tissue-like turbid slab in the nearinfrared spectral range by tomosynthesis. Transmittance data are obtained for a large number of oblique projection directions by scanning a cw laser source across the surface of the slab and by using a CCD camera for spatially resolved light detection. A perturbation model of light transport is used to convert the intensity maps for the different projections into absorption maps. By applying the tomosynthesis approach to these new maps, 3D absorption information on embedded inclusions has been obtained for the first time. The number and the positions of the lateral offset detectors have been optimized by employing a structural similarity index for comparison of the reconstructed with the true absorption data. We present $3 \mathrm{D}$ reconstruction of absorption maps using both Monte Carlo simulations and experiments on phantoms with breast-like optical properties. A comparison with conventional 3D reconstruction by a finite element approach shows the superior location performance of tomosynthesis.
\end{abstract}

\section{Introduction}

Near-infrared (NIR) optical imaging of tissue has been a very active field of research during the past decades. Most of the efforts have been devoted to functional imaging of the brain (Scholkmann et al 2014) and to the detection and characterization of breast cancer (Grosenick et al 2016). Whereas functional brain imaging aims mainly at the detection of changes in blood related parameters like hemoglobin concentration and oxygen saturation, breast imaging and spectroscopy pursue the quantification of tissue absorption and scattering properties. From absorption coefficients at several wavelengths information about the breast tissue composition can be derived (Taroni et al 2017). In particular, the concentrations of oxyand deoxyhemoglobin, and the content of fat, water and collagen can be estimated. Scattering properties yield information about the cellular structure of the tissue. To reliably determine differences between healthy breast tissue and benign alterations on one side and cancerous tissue on the other side, appropriate techniques for spatially resolved data acquisition and analysis are required.
Tomographic geometries provide inherently a three-dimensional set of the breast tissue properties. The tomographic techniques are based on the inversion of the diffusion equation or another model of light propagation in tissue like Monte Carlo simulations (Arridge 2011). Generally, the inversion is time consuming and the resulting three-dimensional (3D) images are blurred due to the strong scattering of NIR light in tissue (Van de Ven et al 2009, Enfield et al 2011). Accordingly, several attempts were undertaken to exploit prior knowledge on the tissue spatial structure in the optical reconstruction derived by conventional breast imaging methods like x-ray mammography (Michaelsen et al 2016, Zimmermann et al 2017, Fang et al 2011), magnetic resonance imaging (MRI) (Mastanduno et al 2015) or ultrasound (Xu et al 2016, Vavadi et al 2018).

Two-dimensional optical scanning of the breast in the parallel plate geometry is another method to obtain optical images of the breast (Grosenick et al 2016). In its simplest configuration, a source and a detector optical fiber are moved in tandem over the breast yielding two-dimensional transillumination images. By using the time-domain or the frequencydomain technique together with a homogeneous 


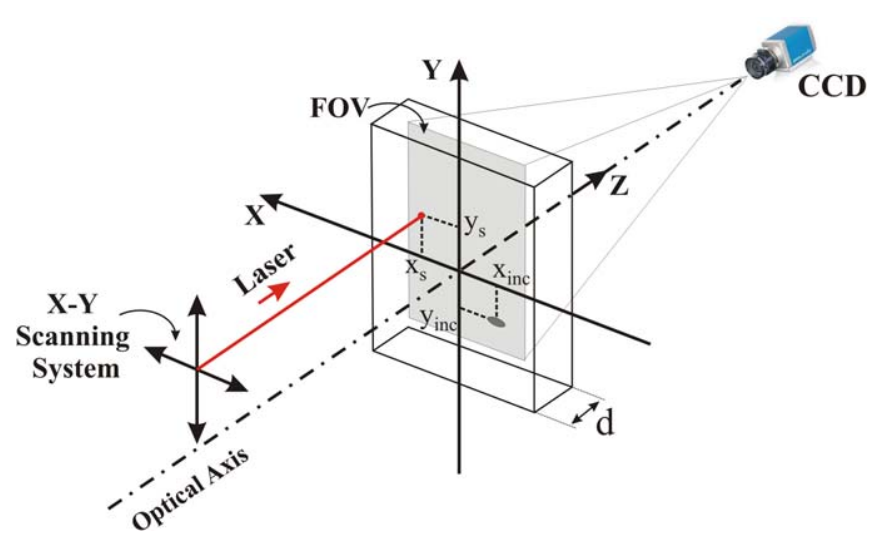

Figure 1. Experimental setup for using a CCD camera as a set of multiple detectors at different offsets relative to the illumination point.

model for data analysis, absorption and reduced scattering coefficients representing average optical properties of the sampled volume can be derived for each scan position (Grosenick et al 2016). To obtain depth resolved information, additional detection fibers have been mounted with fixed offsets around the central detection fiber (Grosenick et al 2004). In this way, oblique projection directions can be realized within limited ranges. Data from such measurements have been analyzed by $3 \mathrm{D}$ reconstruction as well (Dierkes et al 2005). Optical tomosynthesis is an alternative method for obtaining $3 \mathrm{D}$ resolution in the parallel plate geometry. This approach follows the basic concept of X-ray digital breast tomosynthesis (Niklason et al 1997, Wu et al 2004, Poplack et al 2007, Park et al 2007) by combining two-dimensional intensity maps from different projection directions in a simple and fast way. The algorithm has been successfully applied to study tissue absorption and fluorescence on patients with breast cancer (Grosenick et al 2011). These exploratory investigations were done with only four projection directions since the underlying time resolved technique was expensive thereby limiting the number of detection channels. Generally, it is known that the use of CCD cameras with CW sources can improve the information content of measurements due to employing large datasets and an increased field of view (FOV) (Graves et al 2003, Choe et al 2005). Accordingly, a larger number of projection directions is expected to improve the spatial resolution in optical tomosynthesis. However, there are no comprehensive results available on the performance of optical tomosynthesis so far. In the following, we perform a systematic study on contrast and spatial resolution achievable by optical tomosynthesis. To this end, a large range of projection directions in the parallel plate geometry is realized by an EMCCD camera with high dynamic range. Using a structural similarity index (SSIM) to compare the true and the reconstructed geometry we optimize the number of detectors and the required projection angles.

Basic investigations are performed on simulated transmittance data using Monte Carlo simulations for a slab with embedded inclusions. We also make a comparison with a conventional $3 \mathrm{D}$ reconstruction algorithm. In the second part, we successfully demonstrate the application of the technique on phantom measurements. Furthermore, we present a perturbation-based algorithm which enables us to convert the $3 \mathrm{D}$ intensity maps into absorption images.

\section{NIR tomosynthesis of turbid media using a CCD camera}

\subsection{Intensity-based tomosynthesis analysis}

We consider investigations on a slab-like diffuse medium in transmission geometry. Under clinical conditions, this medium could be a human female breast being gently compressed between two transparent parallel plates. For our basic investigations here, the medium is a block-like phantom with tumor simulating inclusions. The experimental setup is presented in figure 1 . The medium of thickness $d$ is illuminated by a laser beam of the proper wavelength in the NIR.

On the exit face of the medium, a EMCCD camera, Andor iXon Ultra 897 is used to record the diffusely transmitted light intensity. Using a EMCCD camera, even for low amplifications settings, greatly improves both, the lower detection limit and the signal to noise ratio, two features that are very desirable for our experiment. The raw data acquisition process consists of scanning the laser over the entrance surface of the medium, and for each position of the laser, a picture is acquired by the camera, which images the desired FOV at the opposite face. Having the complete set of images for all laser positions, the tomosynthesis analysis can be carried out.

Using figure 2, which is an illustration of our problem in one dimension, the basic equations involved 


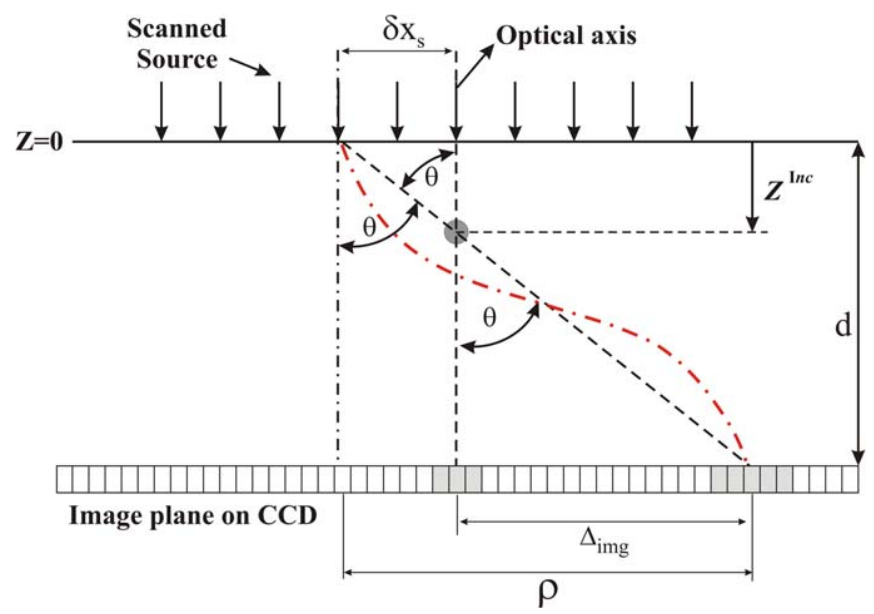

Figure 2. Illustration in one dimension of the CCD approach to the tomosynthesis procedure procedure. An inclusion is considered at depth $Z^{\text {Inc }}$ inside the medium, with $Z=0$ at the face being illuminated by the laser. For each shift of the laser relative to the optical axis, $\delta X_{s}$, a given shift of the image at the CCD plane, $\Delta_{i m g}$, takes place. Note that the offset $\rho$ is, as usual, the lateral distance between source and detection zone. The straight black line (dash) is the pure geometrical path while the red (dash-dot) curve is a representation of the optical path of the photons inside the turbid medium.

in our method can be deduced. Taking as $Z=0$ the plane at which the slab of thickness $d$ is illuminated, for an inclusion located at a depth $Z^{\text {Inc }}$ inside the medium, and using simple geometrical considerations it is easily deduced that

$$
\Delta_{i m g}=\rho \frac{\left(d-Z^{I n c}\right)}{d}
$$

where $\Delta_{i m g}$ is the shift of the image of the inclusion for an offset $\vec{\rho}$ between source and detector compared to on-axis transillumination with the source located in front of the inclusion. Note that the offset is a vector, $\vec{\rho}$, so that it is possible to vary its magnitude as well as it directions on the CCD plane, without the need of repeating the scanning, which constitutes a leading idea of this work.

The purely geometrical shifts of equation (1) need to be corrected for the effects of light propagation inside a diffusive medium. This is done in this work using the most probable photon trajectory approach as described in the paper by Grosenick et al (Grosenick et al 2011) and represented in figure 2 by the red (dashdots) curve.

Briefly described, the proposed algorithm goes through the following main steps:

(i) Scan the whole medium with the laser and capture an image of the exit face for each laser position.

(ii) In each image identify the laser position and integrate the intensity, $I$, in a given area centered at this position. By storing the intensities for all scan positions in a matrix the zero offset image $I$ $(\rho=0)$ is obtained. The area of integration must be chosen accordingly to the experimental conditions and with the following trade-off: it needs to be large enough to capture sufficient light but small enough to keep good spatial resolution. (iii) Define a set of source-detector offsets $\vec{\rho}_{l}$ appropriate for the tomosynthesis algorithm below.

(iv) For each offset vector $\vec{\rho}_{l}$ : in each camera image, shift the area of integration by $\overrightarrow{\rho_{l}}$ with respect to the laser scan position and perform the integration. For each scan position, store the result in the intensity matrix $I\left(\vec{\rho}_{l}\right)$ which represents the offset image for $\vec{\rho}_{l}$.

(v) Define a set of planes $Z_{k}=$ constant and, for each plane, calculate the shift value $\Delta_{i m g}\left(Z_{k}\right)$ as a function of the detector offset $\rho$ using the most probable photon trajectory.

(vi) For each plane $Z_{k}$, shift the intensity images $I\left(\vec{\rho}_{l}\right)$ by the value $\Delta_{i m g}\left(Z_{k}\right)$ as a function of the detector offset $\overrightarrow{\rho_{l}}$ using the most probable photon trajectory.

(vii) Apply one of the tomosynthesis algorithms discussed below to the shifted images $I_{\text {shift }}\left(\vec{\rho}_{l}, Z_{k}\right)$ to construct the $3 \mathrm{D}$ intensity map of the medium.

The algorithm previously presented was written in Python using libraries from NumPy and SciPy. The code of said implementation as used for the present paper is available in GitHub (Carbone).

In the original tomosynthesis approach the shifted images for each plane are simply added. When an inclusion is located in the selected plane, its positions in each shifted image will coincide, and its signal will be increased when the addition is made. On the other hand, if the inclusion is out of the chosen plane, its position will be different for each shifted image and, thus, it will be blurred in the reconstruction. For the present contribution, this addition should be normalized as the number of shifted images used for the reconstruction may be different for each reconstructed pixel. 


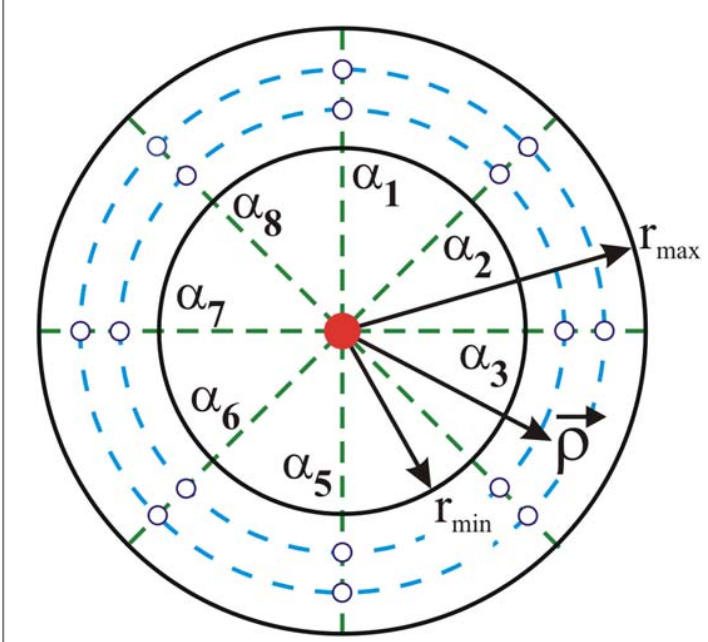

Figure 3. Detector positions (white circles) as a function of maximum and minimum radius, radial step and angular step. The red disc corresponds to the on axis situation. Light intensity is read in a small cluster of pixels $(3 \times 3$ in our case $)$ around each nominal detector position.

Also, as a distinctive feature, the large number of shifted images for each plane $Z_{k}$ obtained by our camera approach gives us the possibility to use several statistical quantities for the tomosynthesis reconstruction. We will consider and compare the following base quantities:

- Average: for each reconstructed pixel, the average of each shifted image is used.

- Median: for each reconstructed pixel, the median of each shifted image is used.

- $20 \%$ percentile: for each pixel, the values within the lowest $20 \%$ of the histogram are used.

- $80 \%$ percentile: for each pixel, the values within the highest $20 \%$ of the histogram are used.

In principle, each pixel of the image captured by the CCD can be used as a detector with its own $\vec{\rho}$. However, given that the computation time of the reconstruction depends on the number of sourcedetector pairs (which would be very big if using all pixels as detectors), in our reconstruction algorithm we chose to use a smaller number of virtual detectors. Also, averaging several pixels around $\vec{\rho}$ allows us to reduce the noise of the final reconstruction.

The number of virtual detectors is a trade-off between noise levels and, at least potentially, reconstruction resolution. As it will be seen, given the diffuse nature of the light's propagation, the resolution saturates with far less detectors than pixels available in the CCD. Nevertheless, even this relatively low number of detectors would be much more costly and complex to implement using individual optical fibers for each of them. Among other advantages, using CCDs allows for a dynamic field of view, faster data acquisition and an overall simpler experimental setup.

Figure 3 shows how these detectors are arranged for a given source position. The red dot represents the position of the source and the hollow circles are the detectors. The number and positions of the detectors are then governed by the following parameters in the reconstruction algorithm:

- $r_{\max }$ and $r_{\text {min }}$ : the maximum and minimum lateral distance $(\rho)$ between the source and each detector.

- the radial step: the distance between rings over which the detectors are located.

- the angular step: the angular distance between detectors within each ring.

This configuration allows most of the computations to be done in the polar space, greatly improving speed and reducing complexity. In the example of figure 3, two detectors rings are shown, and the angular step is $45^{\circ}$ giving a total of 16 detectors. $r_{\max }$ is limited by the amount of light captured and by the geometrical limits of the CCD when the source is located close to the corners of the area of interest. $r_{\text {min }}$ can be as low as 0 , but very low values give poor depth resolutions, as the relative difference in shifts between different planes are too small. The radial and angular steps can be as high as the resolution of the CCD allows, but as we will show in section 4 , the image quality saturates rather quickly with increasing number of detectors.

\subsection{Absorption coefficient recovery}

The tomosynthesis algorithm introduced above can only retrieve relative light intensities. However, if certain assumptions are made, a 3-dimensional map of absorption coefficients can also be derived. If both, the optical properties of the homogeneous medium and the characteristic size of the inclusion are known, and if we assume that the scattering coefficient of the inclusion is approximately the same as that of the surrounding tissue, we can use a perturbation model to calculate the absorption coefficient(s) of the inclusion(s). The Born approximation of the $\mathrm{CW}$ diffusion equation describes the photon density, $u(\vec{r})$, due to small absorption changes, $\Delta \mu_{a}(r)$ (Sassaroli et al 2006):

$$
\begin{aligned}
u(\vec{r})= & u_{0}(\vec{r}) \\
& -\int_{V} G_{0}\left(\vec{r}, \vec{r}^{\prime}\right) \Delta \mu_{a}\left(\vec{r}^{\prime}\right) u_{0}\left(\vec{r}^{\prime}\right) d V,
\end{aligned}
$$

where $u_{0}(\vec{r})$ is the photon density when no perturbations are present, and $G_{0}\left(\vec{r}, \vec{r}^{\prime}\right)$ is the corresponding CW Green's function of the slab.

If we consider that there is a small volume $V$ representing an inclusion with absorption coefficient $\mu_{a}^{I n c}$ present in the medium, it can be shown 
(Sassaroli et al 2006, 2014) that

$$
\frac{\Delta u(\vec{r})}{u_{0}(\vec{r})}=\left\langle l_{I n c}\right\rangle\left(\mu_{a}^{I n c}-\mu_{a}^{0}\right),
$$

being $\frac{\Delta u(\vec{r})}{u_{0}(\vec{r})}$ the relative change in photon density and $\left\langle l_{\text {Inc }}\right\rangle$ the mean pathlength of the detected photons inside the inclusion. We have thus,

$$
\mu_{a_{I n c}}=\mu_{a_{0}}+\frac{1}{\left\langle l_{I n c}\right\rangle} \frac{\Delta u(\vec{r})}{u_{0}(\vec{r})} .
$$

According to the partial-current boundary condition (Contini et al 1997, Haskell et al 1994), the photon densities $u$ and $u_{0}$ are proportional to their derivatives along $\mathrm{z}$ at the boundary of the medium. Hence, the ratio $\frac{\Delta_{u}}{u_{0}}$ can be replaced by the corresponding ratio of the measured intensities. Furthermore, assuming that the inclusion is small enough, the required unperturbed intensity can be approximated by the average of the intensities of each position.

Equation (4) is applied to each intensity image $I\left(\vec{\rho}_{l}\right)$ obtained by step (iv) of the tomosynthesis algorithm described above. The resulting absorption offset images are used then in steps (vi) and (vii) of the algorithm to construct the $3 \mathrm{D}$ absorption maps.

The pathlength inside the inclusion, $\left\langle l_{\text {Inc }}\right\rangle$, is difficult to obtain, as it depends not only on the size of the inclusion but also on its shape. We simplify the inclusion taking it as a 'slab' of the same thickness as the diameter of the actual inclusion. Then, using the analytical expression for the mean time of flight through a slab in transmission (Contini et al 1997) we can approximate $\left\langle l_{\text {Inc }}\right\rangle$.

The required previous knowledge and assumptions may seem excessive, but they can all be obtained by other methods, are usually known or can, in the worst case, be introduced as reasonable guess values.

The optical properties of the homogeneous medium can also be retrieved from the literature for typical tissues or can be measured by e.g. time-resolved techniques. In many interesting clinical cases the reduced scattering coefficient of the inclusion is not very different from the surrounding tissue. However, knowing this parameter is a weak requisite, as good results are obtained even if there are significant differences in the scattering coefficient between inclusion and medium.

Finally, the size and number of inclusions could be known when evaluating a clinical case, for example by previous MRI (Brooksby et al 2004, Cochran et al 2019), Ultrasound (Zhu et al 2003), x-ray tomography (Li et al 2003) or even x-ray tomosynthesis studies (Fang et al 2011). However, our proposal does not require simultaneous recording of optical data combined with any other of these, but only the spatial information that they could provide. Thus, the appropriate clinical scenario for the all optical approach presented in this paper could be to follow the evolution of confirmed lesions with the help of previous geometrical information provided by anyone of the techniques already mentioned. In this way, a two-pass approach can be used, first running a tomosynthesis reconstruction on intensity data, and then using the auxiliary spatial information to retrieve the absorption map. This way will be followed throughout the next sections.

\section{Materials and methods}

We tested our proposal on both, $M C$ simulations and phantom experiments. First we used the MC simulations to evaluate the performance of the proposed approach, and then we show an example of an experiment on a liquid phantom containing two solid inclusions. The geometrical aspects of both are identical and are shown in figure 4.

\subsection{MC simulations}

As a proof of principle, we first considered $M C$ simulations in a diffusive medium with a pair of inclusions. The simulations were performed using the software described in detail in the paper by (Carbone et al 2017). Considering the geometry presented in figure 4 , the source, located at plane $Z=0$, was moved in this plane for each different simulation, scanning an area of $10.4 \times 9.4 \mathrm{~cm}^{2}$ in $X$ and $Y$ direction, respectively, and in $0.2 \mathrm{~cm}$ steps. In each simulation $5 \times 10^{9}$ photons were launched and those succeeding in travelling through the medium were collected on an array of $14 \times 14 \mathrm{~cm}^{2}$ at the exit face, i.e. $Z=5 \mathrm{~cm}$. This size of the collecting array matches the FOV to be used in the phantom experiments. The resulting image had a size of $512 \times 512$ pixels. With the help of a GPU card (Nvidia ${ }^{\circledast}$ GeForce GTX Titan Xp) running under $C U D A$, the total time to complete the simulation for a single source position was about $3 \mathrm{~min}$. The medium was divided into cubical voxels of lateral dimensions $0.1 \times 0.1 \times 0.1 \mathrm{~cm}^{3}$ and the inclusions were defined as a set of voxels with optical properties different from those of the bulk. The host medium and the inclusions were considered to have the same scattering coefficient, namely $\mu_{s}^{0}=50 \mathrm{~cm}^{-1}$ and the same anisotropy factor, $g=0.8$. The reduced scattering coefficient of both the host and inclusions was thus $\mu_{s}^{\prime 0}=\mu_{s}^{\prime I n c}=$ $10 \mathrm{~cm}^{-1}$. The absorption coefficient of the bulk was set to $\mu_{a}^{0}=0.1 \mathrm{~cm}^{-1}$ and we have considered one inclusion more absorbent than the bulk, shown as $I_{1}$ in figure 4 , with $\mu_{a}^{I_{1}}=2 \mu_{a}^{0}=0.2 \mathrm{~cm}^{-1}$, and one inclusion less absorbent than the bulk, namely $I_{2}$ with $\mu_{a}^{I_{2}}=0.5 \mu_{a}^{0}=0.05 \mathrm{~cm}^{-1}$. Both inclusions consisted of spheres, conformed by 522 cubical voxels, with a diameter of $1 \mathrm{~cm} . I_{1}$ was located at $\left(x_{1}^{I n c}, y_{1}^{I n c}, z_{1}^{I n c}\right)=$ $(+1.25,+1.10,+1.25) \mathrm{cm}$, and $I_{2}$ was placed at $\left(x_{2}^{I n c}, y_{2}^{I n c}, z_{2}^{I n c}\right)=(-1.25,-1.10,+3.75) \mathrm{cm}$.

Please note that the selected positions of the inclusions represent challenging ones since they lay deep inside the medium, and at depths where the actual photon paths deviate the most from the simple geometrical 


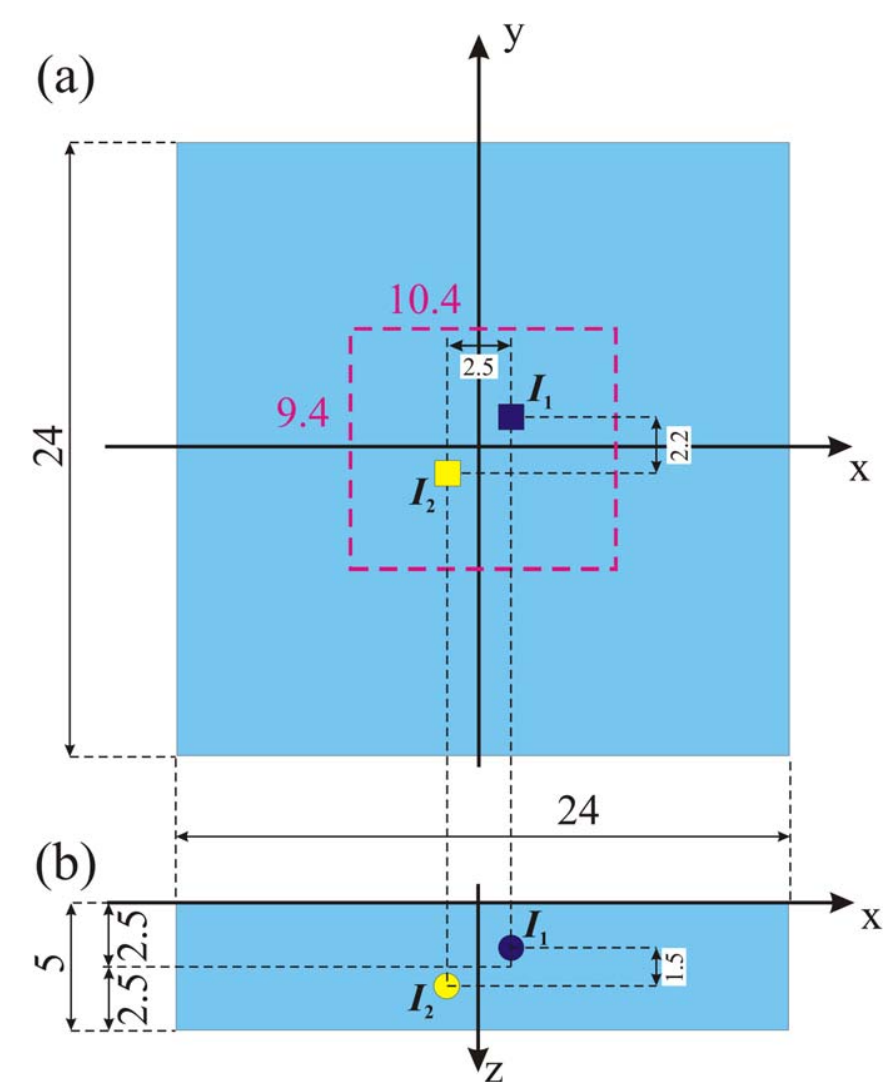

Figure 4. Geometrical aspects of the MC simulations and experiment. The dashed red rectangle enclosures the area scanned by the source. The optical axis is at point $(x, y)=(0,0)$. (a) Camera view, and (b) Top view. All dimensions in $\mathrm{cm}$.

one, thus maximizing the effects of diffusion, as seen in figure 2 . A total of $52 \times 47=2444$ source positions were simulated; these will be also used in the experiments described later.

\subsection{Phantom experiments}

For the experiments on phantoms we used the same geometry of figure 4, but with two identical absorbing inclusions. The host consisted of a cuvette filled with a solution of milk, water and India ink in the right proportions to produce optical properties similar to breast tissue. The optical properties were obtained by fitting the resulting distributions of time of flight (DTOF) acquired by time-resolved measurements to the model described by Contini et al (Contini et al 1997, Pardini et al 2015). They resulted: $\mu_{a}^{0}=$ $0.07 \pm 0.01 \mathrm{~cm}^{-1}$ for the absorption coefficient and $\mu_{s}^{\prime 0}=8.9 \pm 0.7 \mathrm{~cm}^{-1}$ for the reduced scattering coefficient. The cuvette was $5 \mathrm{~cm}$ thick, and its lateral dimensions were $24 \times 24 \mathrm{~cm}^{2}$. Immersed in this host medium, two absorbing inclusions were placed. They were also made from a mixture of milk, water and India ink with added agarose to provide solidification and consisted of cylinders with a diameter of $1 \mathrm{~cm}$ and a height of $1 \mathrm{~cm}$ each.

To construct these inclusions we first made a slab using the mixture of water, milk, India ink and agarose (2\% in volume) described above. This slab had $10 \times 10 \mathrm{~cm}^{2}$ lateral dimensions and was $3 \mathrm{~cm}$ thick.
We then measured their optical properties also by fitting the DTOF resulting from time resolved measurements and obtained: $\mu_{a}^{I n c}=0.26 \pm 0.03 \mathrm{~cm}^{-1}$ for the absorption coefficient and $\mu_{s}^{\prime I n c}=8.2 \pm 0.7 \mathrm{~cm}^{-1}$ for the reduced scattering coefficient. After that, the inclusions were extracted from this slab by 'cutting' them out with a cylindrical plastic tube of $1 \mathrm{~cm}$ diameter and were finally trimmed to the desired length of $1 \mathrm{~cm}$.

Inclusion $I_{1}$ was located at $\left(x_{1}^{I n c}, y_{1}^{I n c}, z_{1}^{I n c}\right)=$ $(+1.25,+1.10,+1.25) \mathrm{cm}$, and $I_{2}$ was placed at $\left(x_{2}^{I n c}, y_{2}^{I n c}, z_{2}^{I n c}\right)=(-1.25,-1.10,+3.75) \mathrm{cm}$. Both inclusions were mounted inside the cuvette previously to filling it with the milky solution. They were held in place by means of a spanned thin thread going through the cylinders and fixed to the bottom and the top of the cuvette. Please note that because of this procedure the locations of the inclusions given above are nominal and the actual ones can slightly differ from them.

The illumination source was a diode laser operating at $\lambda=785 \mathrm{~nm}$, and $5 \mathrm{~mW}$ average power. The FOV of the CCD camera (Andor iXon Ultra 897, EMCCD) was an area of the exit face of the phantom of $14 \times 14 \mathrm{~cm}^{2}$, which was imaged onto an array of $512 \times 512$ pixels as already schematized in figure 1 . An optical fiber connected to the laser diode was mounted on a 2D gantry system (Zaber Technologies T-G-LSM200A), and its distal end was kept in contact with the front face of the cuvette allowing to accurately 


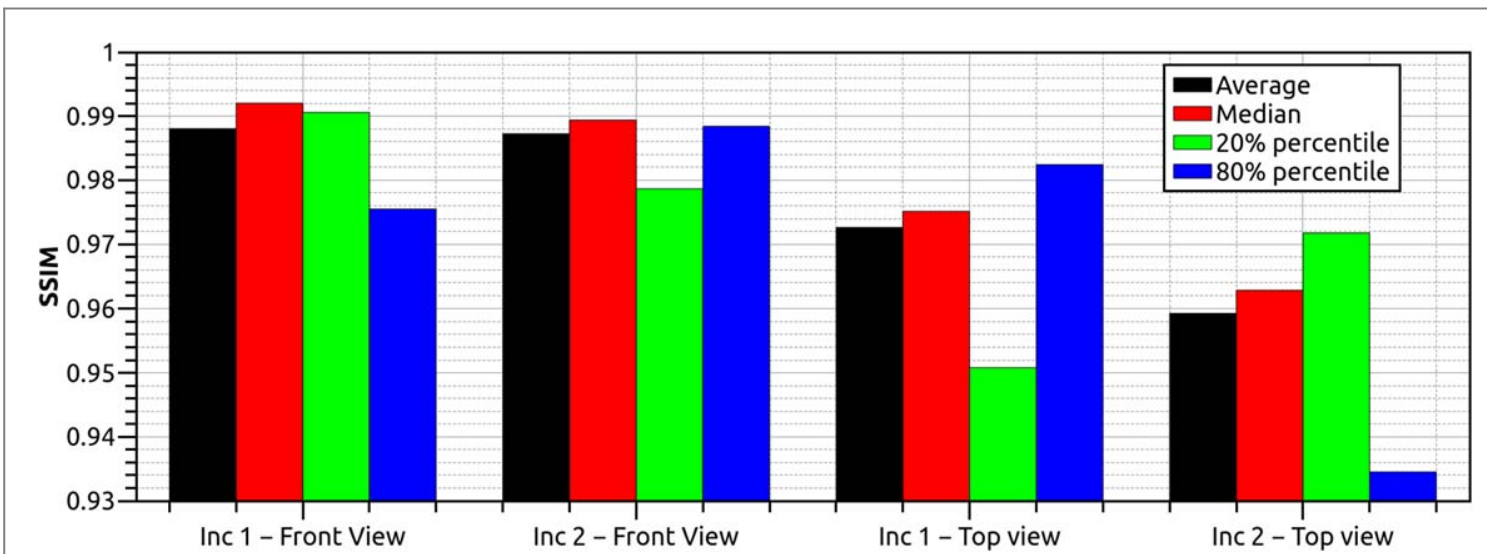

Figure 5. Structural Similarity Index for each reconstruction method and for both inclusions. The legend Front View corresponds to a slice in the $Z=$ constant plane, while Top View indicates a slice in the $Y=$ constant plane.

position the emitting source on the entry face of the phantom. As in the previous case of MC simulations, an array of $52 \times 47=2444$ source positions covering an area of $10.4 \times 9.4 \mathrm{~cm}^{2}$ was used.

\section{Assessment of algorithm performance}

Using the MC simulations described in section 3.1 we performed different reconstructions changing both, the method for combining the individual images and the number of detectors. This last parameter was chosen by selecting the number of detector rings and the number of angular positions according to figure 3 .

To assess the quality of the reconstructions, we compared the resulting images in four selected slices, namely two $Y=$ constant and two $Z=$ constant cuts, located at the center of each inclusion, with a reference, 'perfect', image built using the information of the size and position of the inclusions applied in the MC simulations. To quantify the similarity between the reconstructions and the reference image, we computed the Structural Similarity (SSIM) index between them.

In recent years the SSIM index has become an accepted standard among image quality metrics (Wang et al 1995, Brunet et al 2011, Dosselmann and Yang 2011). Made up of three components, this technique assesses the visual impact of changes in image luminance, contrast and structure. The SSIM index is used for measuring the similarity between two images and is a full reference metric; in other words, the measurement or prediction of image quality is based on an initial reference image. This index is designed to improve other traditional methods such as peak signal-to-noise ratio (PSNR), L1-norm and mean squared error (MSE).

Briefly described, the SSIM index is calculated on a sliding window of an image. For these windows, $x$ from image 1 and $y$ from image 2 of common size $N \times N$, it is defined as:

$$
\begin{aligned}
\operatorname{SSIM}(x, y) & =\frac{\left(2 \mu_{x} \mu_{y}+c_{1}\right)\left(2 \sigma_{x y}+c_{2}\right)}{\left(\mu_{x}^{2}+\mu_{y}^{2}+c_{1}\right)\left(\sigma_{x}^{2}+\sigma_{y}^{2}+c_{2}\right)}, \\
c_{1} & =\left(k_{1} L\right)^{2}, c_{2}=\left(k_{2} L\right)^{2}
\end{aligned}
$$

where $\mu_{x}$ and $\mu_{y}$ are the average intensities of regions $x$ and $y$, respectively; $\sigma_{x}^{2}$ and $\sigma_{y}^{2}$ are the variance of the intensities $x$ and $y$ respectively, and $\sigma_{x y}$ is the covariance of $x$ and $y . L$ is the dynamic range of the possible values of the pixels and $k_{1}$ and $k_{2}$ are constants to stabilize the division.

The resultant SSIM index for two given images is the mean of the SSIMs for each window and is a decimal value between -1 and 1 . The value 1 is only reachable in the case of two identical sets of data and therefore indicates perfect structural similarity. A value of 0 indicates no structural similarity.

For the present contribution we used the implementation of the SSIM algorithm offered by the scikit - image Python package (image development team) with its default configuration: $k_{1}=0.01$, $k_{2}=0.03$ and $N=7$.

The main advantage of using SSIM over other, more standard, metrics is that it is less sensible to pixel noise ('salt and pepper'). This is an important consideration for the present case, as the reconstructed image can be noisy and the signal-to-noise ratio may not be constant between different reconstruction methods. In other words, SSIM attempts to model the perceived change in the structural information of the image, whereas metrics like MSE are actually estimating the perceived errors (Rosebrock).

Nevertheless, we include MSE differences as a comparison.

\subsection{Comparison of tomosynthesis base quantities}

As discussed in section 2, we tried four different base quantities for the tomosynthesis: average, median, $20 \%$ percentile, and $80 \%$ percentile. Figure 5 shows a comparison of the SSIMs for the four methods in the four selected slices using absorption data. For comparison, Figure 6 shows MSEs instead of SSIMs. The 


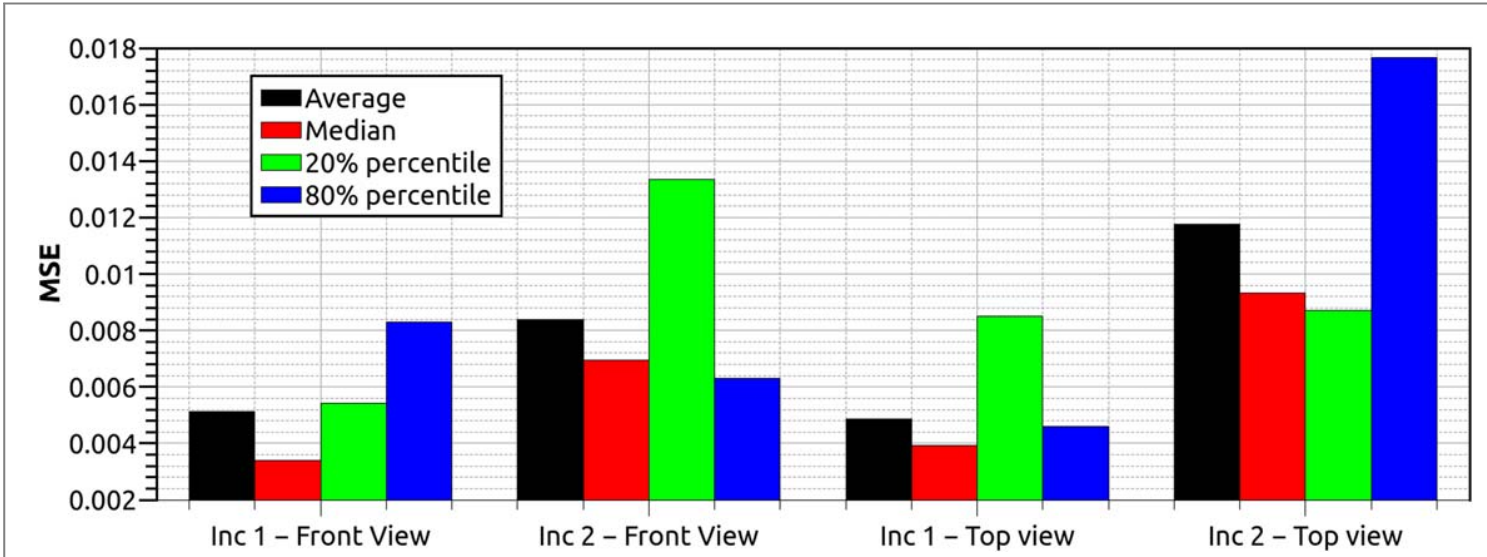

Figure 6. MSE for each reconstruction method and for both inclusions. The legend Front View corresponds to a slice in the $Z=$ constant plane, while Top View indicates a slice in the $Y=$ constant plane.

reconstructions were done with a high number of detectors, by choosing 10 rings and 16 angles, $r_{\min }=1.5 \mathrm{~cm}$ and $r_{\max }=2.5 \mathrm{~cm}$. However, we will show below that these numbers of rings and angles are more than enough for a very good reconstruction, so that the deterioration of the images due to these two parameters can be considered negligible for the analysis within this section.

In all cases the SSIM resulted over 0.92 showing an overall good reconstruction. Average and median have a very similar behaviour, with slightly better values for median specially for the $Y=$ constant slices, thus suggesting a better depth resolution when using median.

The percentile cases are particularly interesting. They show that using percentiles can allow for better depth reconstruction, but only if the general absorption characteristics of the inclusion are previously known, i.e., if it is more or less absorbent than the surrounding host.

This can be understood from the mathematics of the percentiles: for example, for the case of the $80 \%$ percentile, it selects the highest absorption values, effectively enhancing the inclusion if it is more absorbing than the host. Thus, the $80 \%$ percentile achieves very good depth resolution for Inclusion 1 , which is more absorbing than the medium. An analog reasoning can be used for the values within the lower $20 \%$ percentile and a less absorbing inclusion. However, they both have poor depth resolution with the other type of inclusion.

Similarly, the same effect affects the the X-Y resolution as seen in the Front View. In this case, however, both the SSIM and the MSE show better results for the $20 \%$ percentile for Inclusion 1 and for the $80 \%$ percentile for Inclusion 2. This is due to the fact that the better depth reconstruction of the other inclusion allows for a clearer image in the Front View images.

This can be better understood with the help of the figures 7 and 8, where we show the reconstructions on the slices where the inclusions are to be found, using each method. We can see that, overall, (and specially if it is not know if the inclusion is more or less absorbent than the medium) the median achieves better images in agreeing with the previous analysis.

In summary, assuming a general case in which the absorption characteristics of the inclusion(s) are unknown a priori, unless otherwise stated, for the rest of the discussion we will use the median method, since it gives the best overall performance for the reconstruction.

\subsection{Number of detectors}

To evaluate the influence of the number of detectors on the quality of the reconstructed absorption data, we run the algorithm with different numbers of detector rings and angles. The maximum number of angular positions was set to $16, r_{\min }$ was set to $1.5 \mathrm{~cm}$ and $r_{\max }$ to $2.5 \mathrm{~cm}$.

The step between angular positions was set to $\frac{360^{\circ}}{N_{a}}$, where $N_{a}$ is the number of angular detectors. The value of $r_{\min }$ was chosen to be as small as possible and so that it does not overlap with the on axis 'detector'; $r_{\text {max }}$ was selected as the maximum offset compatible with the size of the imaged area.

Figure 9 shows the SSIM calculated at $Y=$ constant of the less absorbing inclusion $I_{2}$ for different number of detector rings, and using two numbers of angular positions, namely 16 (grey dots) and 4 (red dots). This slice was chosen as representative for the other cases as it has the lowest (worst situation) SSIM, as already shown. The results for the other slices are consistent with this one and are not shown for simplicity.

It can be seen from figure 9 that the reconstruction quality improves when the number of detector rings increases, as is expected. However it is quickly asymptotic, showing that increasing the number of rings beyond 7 doesn't improve image reconstruction any further.

Similarly, figure 10 shows the SSIM for different numbers of angular detector positions and for two different numbers of detector rings. As in the previous 


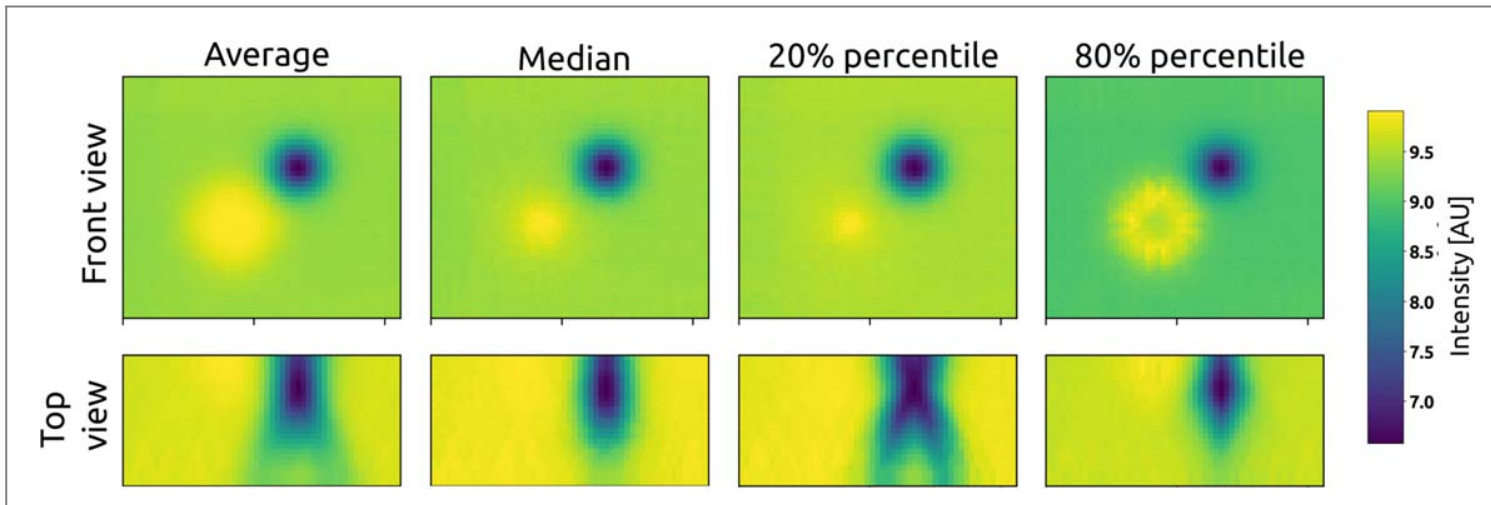

Figure 7. Comparison of the reconstructed images for each of the proposed methods. Inclusion 1
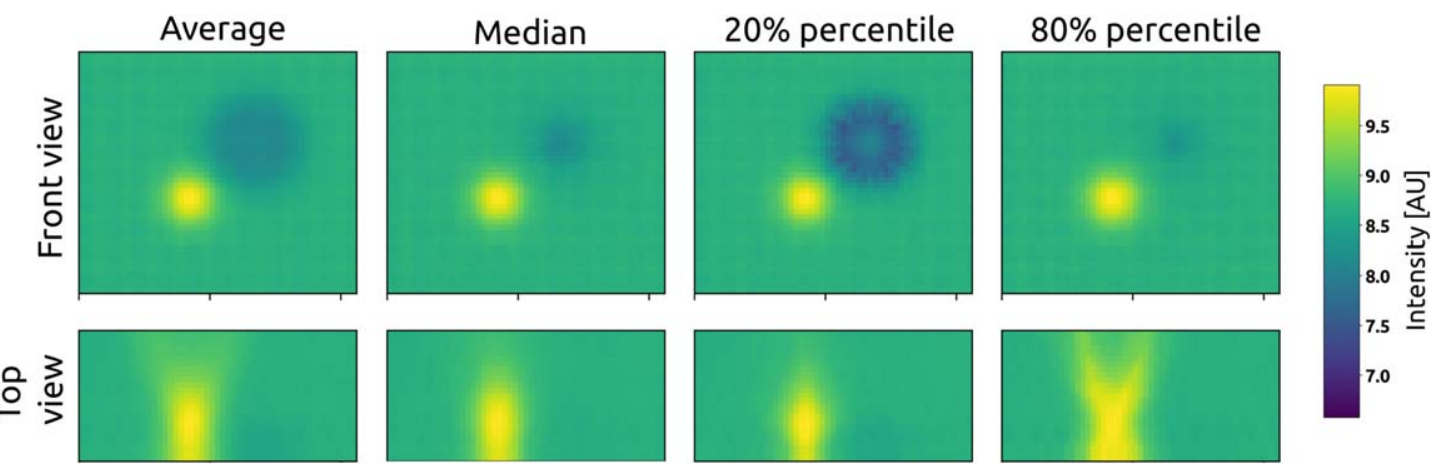

Figure 8. Comparison of the reconstructed images for each of the proposed methods. Inclusion 2.

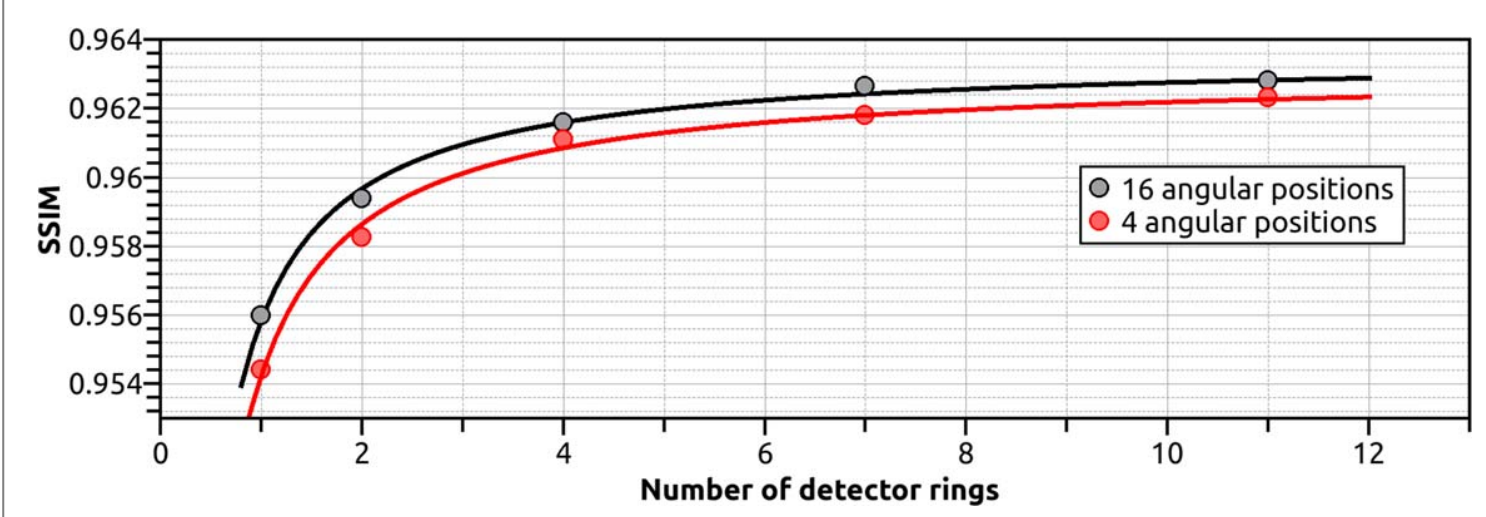

Figure 9. Structural similarity index as a function of the number of detector rings and for two different numbers of angular positions. The solid lines corresponds to an asymptotic function drawn to guide the reader's eye.

case, the $Y=$ constant slice of inclusion $I_{2}$ was chosen as representative but other cases match this behaviour. For this test, the number of radial rings was chosen to be 11 (grey dots) and 4 (red dots), $r_{\min }=1.5 \mathrm{~cm}$ and $r_{\max }=2.5 \mathrm{~cm}$.

Again, though the quality of the reconstruction improves with the number of angular positions, it is quickly asymptotic. These results show that a number of angular positions of about 8 , corresponding to an angular step of $45^{\circ}$, are enough for a good reconstruction.

Another effect of the number of detectors is computation time, which is linear with total number of source-detector pairs. Using the recommended settings of 7 detector rings and 8 angular positions (and thus, 56 detectors per source) and for the 2444 source positions available both in the MC simulations and in the phantom experiments, the full reconstruction takes around $5 \mathrm{~min}$ in an average modern laptop with a 2-core/4-threads CPU using Python and Numpy.

\section{Reconstruction of the absorption maps}

In this section we show the reconstruction of the absorption maps corresponding to both, the $\mathrm{MC}$ 


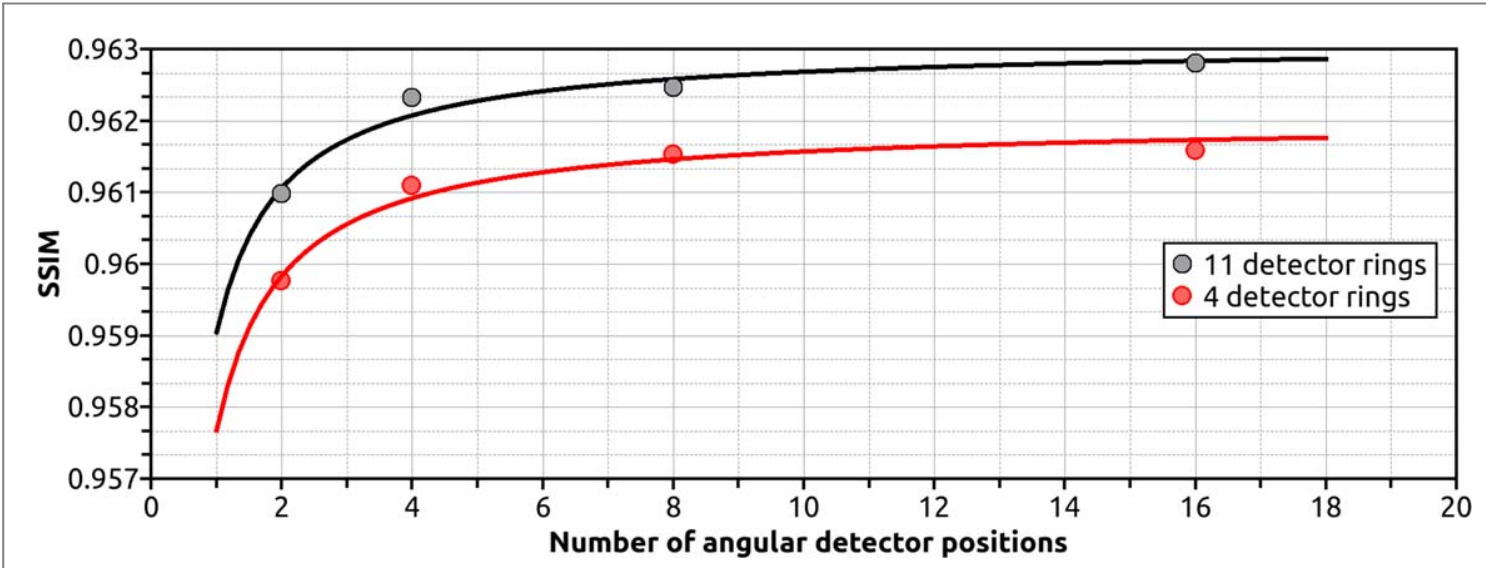

Figure 10. Structural similarity index as a function of the number of detector angles. The step between angles was chosen as $\frac{360^{\circ}}{N_{a}}$, where $N_{a}$ is the number of angular detectors. The solid lines corresponds to an asymptotic function drawn to guide the reader's eye.

simulations and the experiment. The algorithm automatically generates a set of observation planes (or slices). To illustrate the procedure, and for the sake of brevity, we have chosen only a set of 6 slices, $Z=$ constant, from $z=0.4 \mathrm{~cm}$ to $z=4.4 \mathrm{~cm}$, which represent planes normal to the optical axis, but this can be modified at will. Moreover, also planes $X=$ constant or $Y=$ constant can be reconstructed. Please refer to the geometry shown in figure 4.

Consistent with the findings in the previous sections, the reconstruction uses the median of each group of shifted absorption images, the number of rings was set to 7 , the selected number of angular detectors was $8, r_{\min }$ was $1.5 \mathrm{~cm}$ and $r_{\text {max }}$ was $2.5 \mathrm{~cm}$.

Since for MC simulations the optical properties of both, bulk and inclusions, as well as their locations are exactly known (by construction), the performance of the present proposal will be first tested using these simulations. Later on, the results for a phantom experiment will be shown.

\subsection{Absorption reconstruction from simulated data sets}

\subsubsection{Estimation of the size of the inclusions}

The first step for obtaining the absorption map is to recover the intensity maps (or slices), from which the inclusion size can be estimated; then, using these maps together with the size of the inclusions, the procedure described in section 2.2 is used to obtain a map of the (relative) absorption, which is the main goal.

Please note that because of diffusion the inferred size of the inclusions is always overestimated, and thus their absorption results underestimated. Prior knowledge about size can be used to improve the reconstruction of the absorption map, as is shown at the end of this Subsection, using the actual real size of the inclusions, which are given as input in the simulations. In a clinical case, this information could be provided, for example, by an X-Ray or MRI image.
We show in figure 11(a) a set of reconstructed intensity maps for slices in planes $Z=$ constant, which cut the medium parallel to its entrance and exit faces (see figure 4). The coordinates of maximum and minimum absorption from the $3 \mathrm{D}$ data set were taken as the center coordinates of inclusion $I_{1}$ and $I_{2}$, respectively. The corresponding $\mathrm{Z}$ planes are at $z_{1}=1.2 \mathrm{~cm}$ and $z_{2}=3.6 \mathrm{~cm}$. The red circles shown in these planes give the actual position and size, while the black circles show the retrieved positions and sizes. To estimate the sizes of the inclusions from the reconstructed slices we first took intensity profiles along $x$ direction through the center of each inclusion. These profiles, represented by black dots in figures 11(b) and (c), were then fitted to a Gaussian function (red solid curves in figures 11(b) and (c)) from which we inferred their diameters, $\phi$, as the corresponding FWHM of the fitted function assuming that the inclusions are spherically symmetric. The retrieved values are $\phi_{1}=1.35 \mathrm{~cm}$ for inclusion $I_{1}$ and $\phi_{2}=1.28 \mathrm{~cm}$ for inclusion $I_{2}$.

In a similar way as described for figures 11,12 (a) presents a set of selected $X=$ Constant planes. Again, the red circles shown give the actual position and size of the inclusions, while the black circles show the corresponding retrieved ones. Figure 12(b) shows profiles (dots) taken along the $Z$ direction following the vertical black lines shown in the slices for each inclusion. A Gaussian fit of these profiles is also given (red solid line). Though these profiles are smooth and extended along the depth of the slab, the positions of the inclusions is fairly good obtained. Slices and profiles for planes $Y=$ Constant are completely equivalent and it is thus not worth to show them.

\subsubsection{Reconstruction of the absorption coefficient}

After estimation of size and position of each inclusion has been accomplished, we followed the procedure presented in section 2.2 to transform the intensity maps into absorption maps. Figure 13 shows the absorption map retrieved for $Z=$ constant planes, 
a)

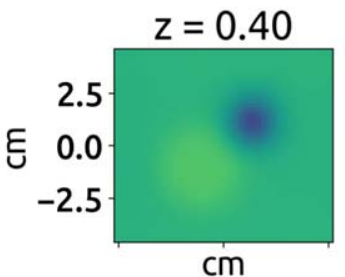

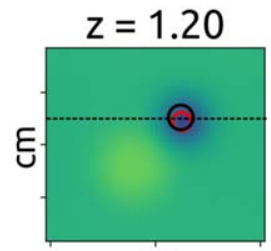

$\mathrm{cm}$

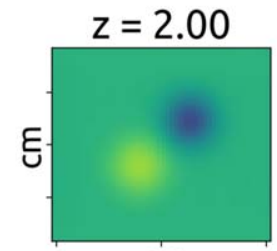

$\mathrm{cm}$

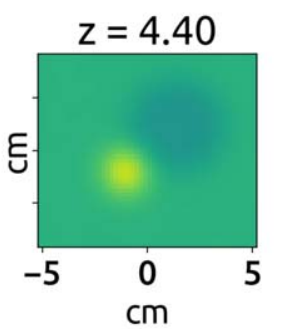

10

9

9

8 논

文

b)
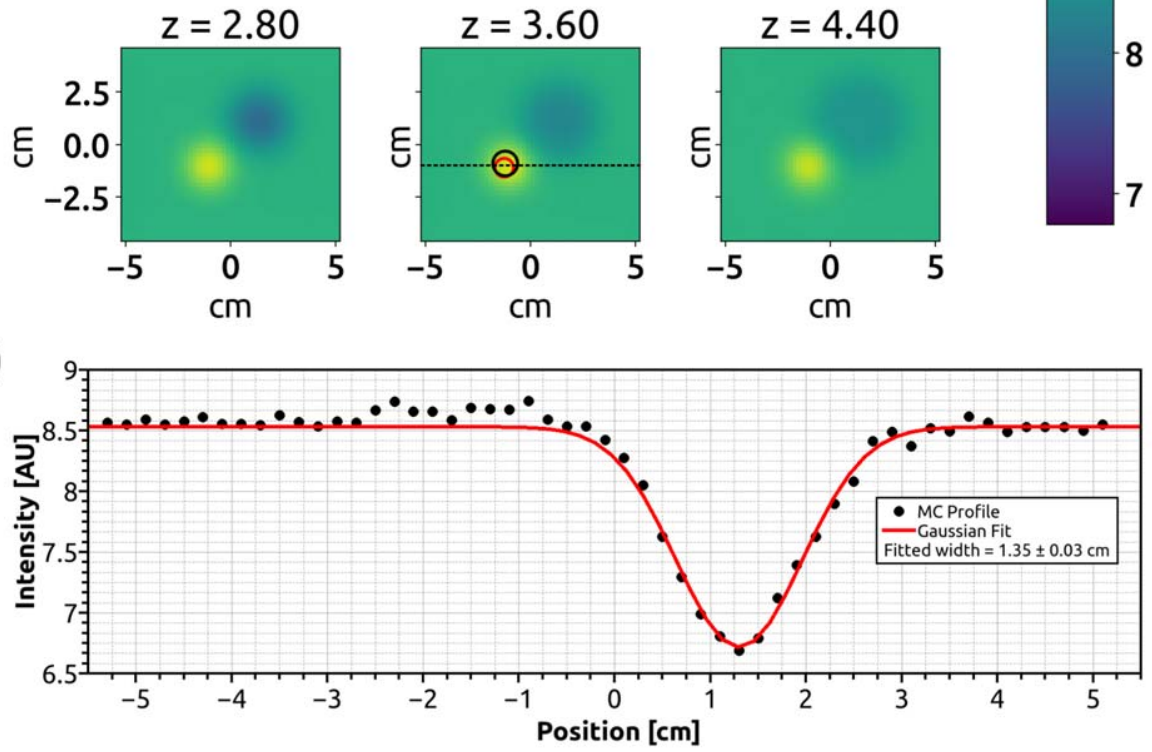

c)

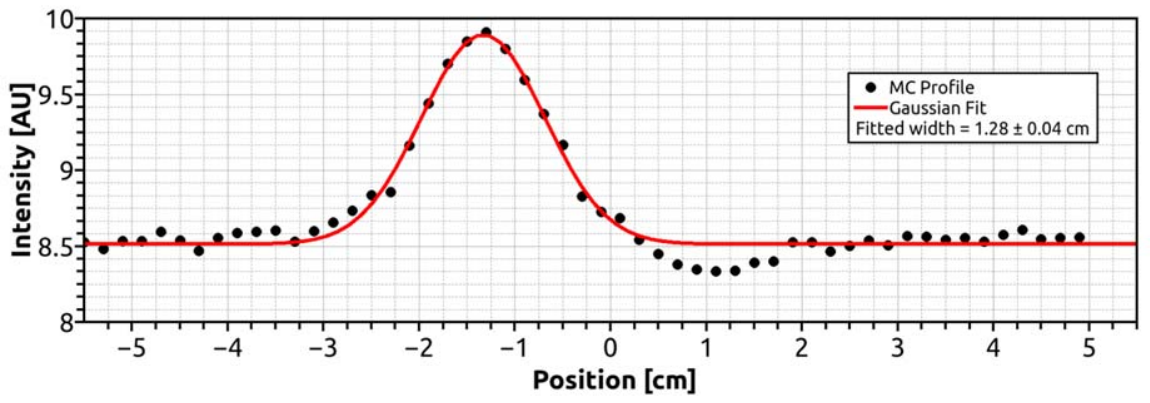

Figure 11. a) Selected slices of the reconstructed 3D intensity maps of the MC simulations corresponding to $Z=$ constant planes. Maximum modulation occurs at $z=1.2 \mathrm{~cm}$ for $I_{1}$ and at $z=3.6 \mathrm{~cm}$ for $I_{2}$. b) Profile (dots) taken along the horizontal black dashed line shown in a) for $z=1.20 \mathrm{~cm}, I_{1}$ (slice at plane $Z=1.2 \mathrm{~cm}$ ) and the corresponding fit to a Gaussian function (solid red). c) Same as b) but for the less absorbing inclusion, $I_{2}$.

using the size of the inclusion inferred from the intensity maps. For comparison, Figure 14 and Figure 15 give the result when the true size of the inclusions is used in the analysis. Table 1 summarizes the nominal and the retrieved parameters.

In figure 13 , the absorption coefficient of inclusion $I_{1}$ is underestimated by $25 \%$, and the absorption of inclusion $I_{2}$ is overestimated by $56 \%$. The main reason for these deviations is the overestimated half width of the inclusions. Accordingly, when exploiting the true size (figure 14), the absorption coefficients are much closer to the true values. For the high absorbing sphere the deviation is only $-10 \%$, for the less absorbing sphere it is $+4 \%$ then. The $\mathrm{Z}$ position deviates by $1.5 \mathrm{~mm}$ (inclusion $I_{1}$ ) and $2.4 \mathrm{~mm}$ (inclusion $I_{2}$ ) which is small compared to the thickness of the slab.
To have at single glance a picture of the results of the whole reconstruction, we present in table 1 the complete set of parameters involved, both, nominal and retrieved.

\subsection{Absorption reconstruction for the phantom experiment}

Similar to the MC simulation, the reconstruction algorithm was applied to the experimental data obtained as detailed in section 3.2. Figure 16 and 17 show absorption results for six slices with $Z=$ constant.

As in the case of the MC simulations, the algorithm is able to recover useful $3 D$ information. The positions and sizes of the inclusions, as seen from the camera, are now marked with red and black squares, since for the experiments small cylinders were used, 
a)
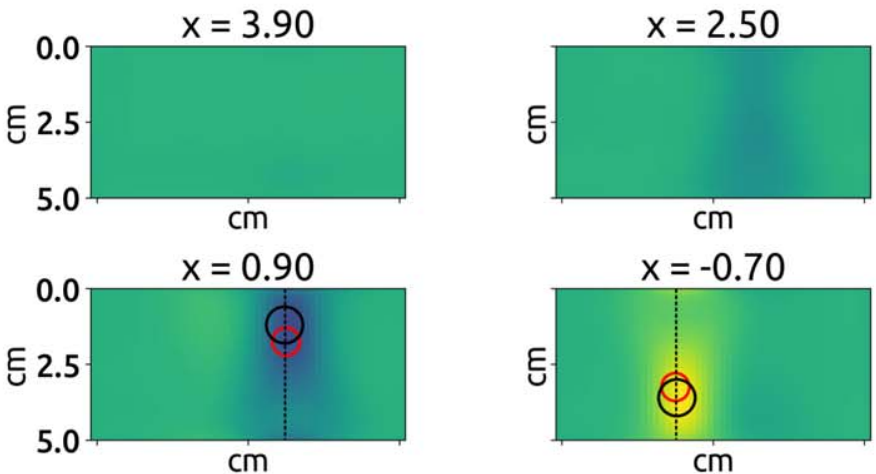

$\mathrm{cm}$
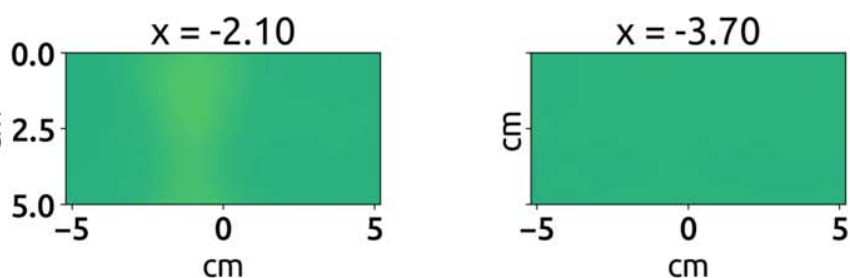

b)
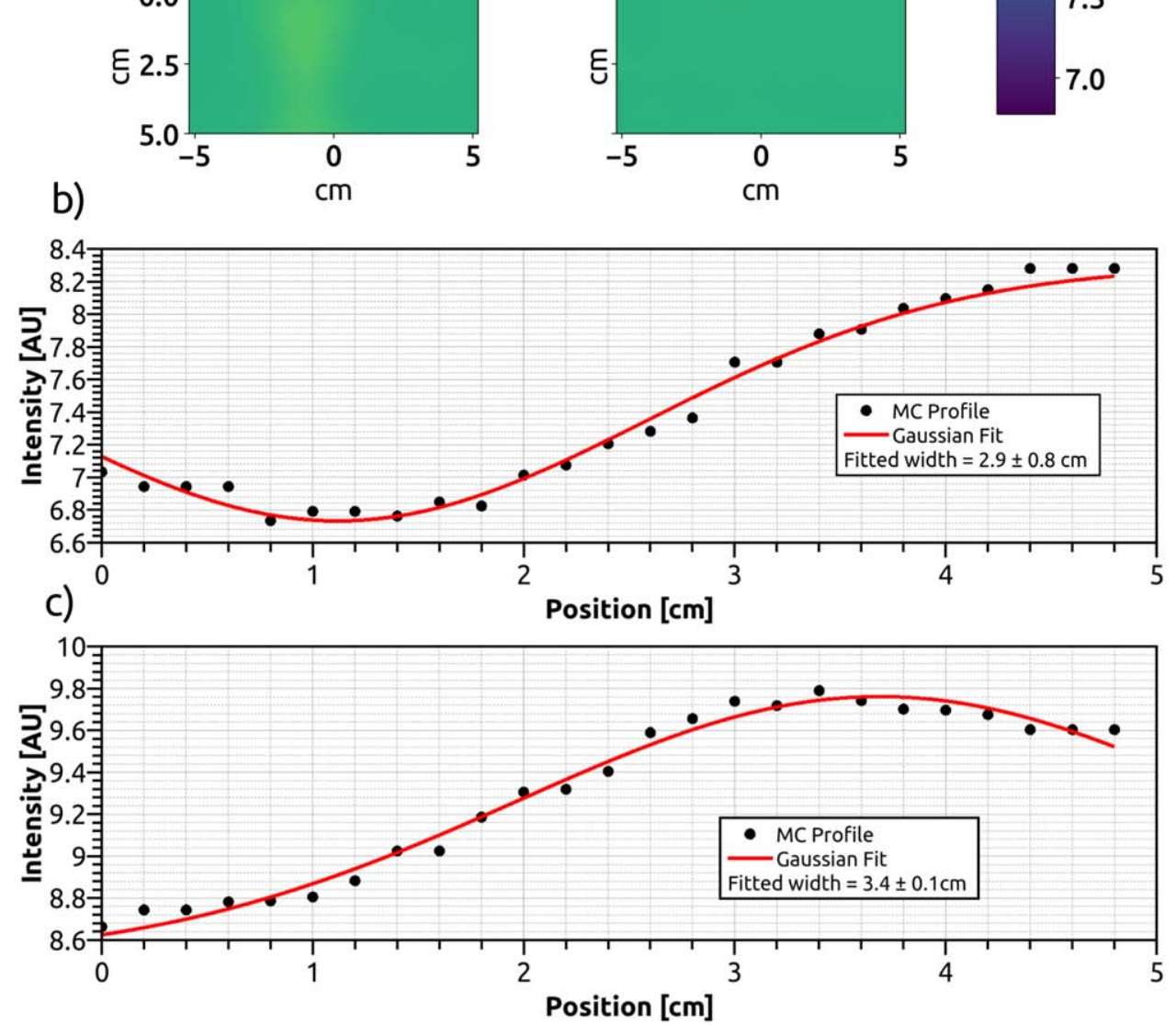

Figure 12. Same as figure 11, but for $X=$ constant planes.

instead of the spheres used for MC. Furthermore, both inclusions have higher absorption now. In table 2 we present a summary of the results for the experimental situation.

For this experimental case, the reconstruction is not as good as in the MC simulations and the absorption coefficient is, overestimation of the size of the inclusion (now 50\% error) compared to the MC simulation. When taking the true size as prior knowledge into account, the deviation reduces to $16 \%$ which is a good result for experimental conditions. A comparison of figures 13 and 16 shows that the inclusions appear more blurred along the $\mathrm{Z}$ direction in the experiment compared to the simulation. Generally, there are a number of different reasons for the limited accuracy in the phantom experiment: noisier input images, uncertainty in the medium and/or inclusion optical properties, uncertainty in the geometry of the inclusions. Nevertheless, the reconstruction is good given the simplicity of the approach and the limits imposed by the diffusive propagation. It is important to remark that both the geometry and the optical properties of the medium and the inclusions are realistic considering possible clinical situations.

\subsection{Comparison with other approaches}

We present in this section a comparison of the performance of our proposal to other algorithms and approaches. In particular we show two comparisons, namely i) to an inversion made with TOAST ++ and 

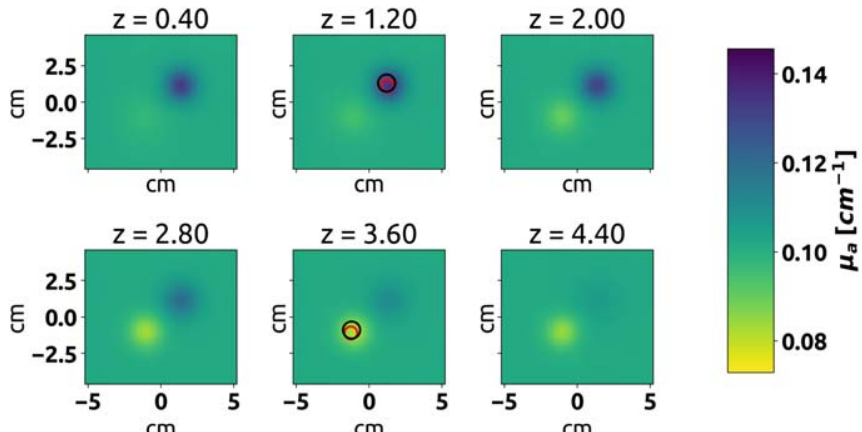

Figure 13. Reconstructed absorption coefficient for six selected $Z=$ constant planes for MC simulations. At planes of maximum modulation for each inclusion, namely $z=1.2 \mathrm{~cm}$ for $I_{1}$ and $z=3.6 \mathrm{~cm}$ for $I_{2}$, the red circles represent the actual position and size of the inclusions. The black circles correspond to the position and size retrieved as shown in figure 11(b).
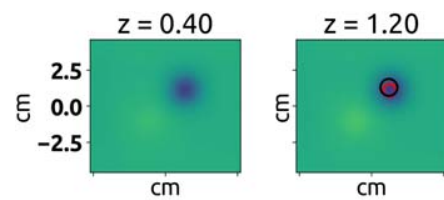

$\mathrm{cm}$
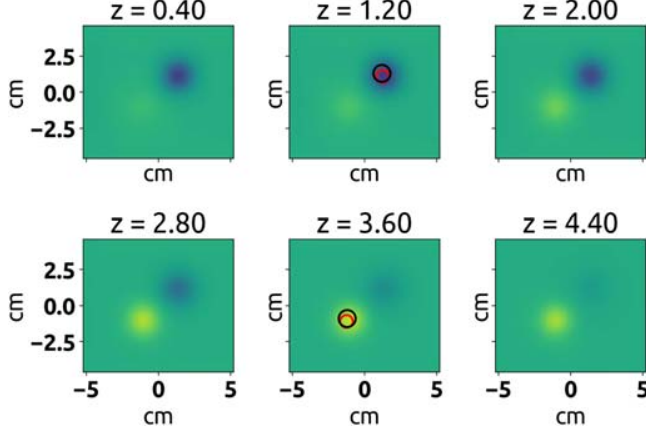

$\mathrm{cm}$
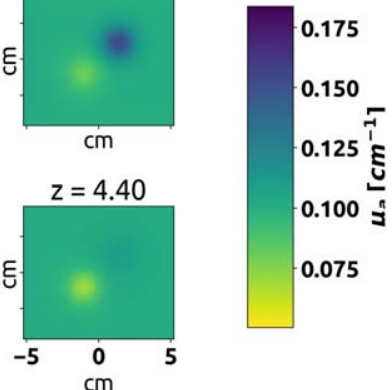

Figure 14. Same as figure 13 but using the nominal sizes of the inclusions. Note that the retrieved absorption coefficients have now values which are closer to the actual ones. $\mu_{a}^{I_{1}}=2 \mu_{a}^{0}=0.2 \mathrm{~cm}^{-1}$ and $\mu_{a}^{I_{2}}=0.5 \mu_{a}^{0}=0.05 \mathrm{~cm}^{-1}$.

Table 1. Summary of the nominal and retrieved parameters, namely depth, diameter and absorption coefficient, for both inclusions in the MC simulations.

\begin{tabular}{|c|c|c|c|}
\hline MC Simulations & & Inclusion 1 & Inclusion 2 \\
\hline \multirow[t]{4}{*}{ Real inclusion } & Nominal position & $(1.25,1.10,1.25) \mathrm{cm}$ & $(-1.25,-1.10,3.75) \mathrm{cm}$ \\
\hline & Diameter & $1 \mathrm{~cm}$ & $1 \mathrm{~cm}$ \\
\hline & $\langle t\rangle$ & $0.105 \mathrm{~ns}$ & $0.134 \mathrm{~ns}$ \\
\hline & Absorption & $0.2 \mathrm{~cm}^{-1}$ & $0.05 \mathrm{~cm}^{-1}$ \\
\hline \multirow[t]{6}{*}{ Tomosynthesis of intensity data } & Retrieved position & $(1.29,1.23,1.2) \mathrm{cm}$ & $(-1.22,-0.95,3.6) \mathrm{cm}$ \\
\hline & Rel. error & $(3 \%, 11 \%, 4 \%)$ & $(10 \%, 13 \%, 4 \%)$ \\
\hline & FWHM & $1.35 \mathrm{~cm}$ & $1.28 \mathrm{~cm}$ \\
\hline & Rel. error FWHM & $35 \%$ & $28 \%$ \\
\hline & $\langle t\rangle$ & $0.165 \mathrm{~ns}$ & $0.224 \mathrm{~ns}$ \\
\hline & Rel. error $\langle t\rangle$ & $57 \%$ & $67 \%$ \\
\hline Absorption using estimated size & & $0.15 \mathrm{~cm}^{-1}$ & $0.078 \mathrm{~cm}^{-1}$ \\
\hline Relative deviation & & $-25 \%$ & $56 \%$ \\
\hline Absorption using real size & & $0.18 \mathrm{~cm}^{-1}$ & $0.052 \mathrm{~cm}^{-1}$ \\
\hline Relative deviation & & $-10 \%$ & $4 \%$ \\
\hline
\end{tabular}

ii) to a recent publication using DOT with simultaneous MRI information.

\subsubsection{Comparison with TOAST ++ reconstructions}

TOAST ++ is a software package for forward and inverse calculations based on a finite element approach for the diffusion equation of photon transport (Schweiger and Arridge 2014). We applied this software to reconstruct the absorption coefficient from the raw intensity data of our MC simulations. The reduced scattering coefficient was fixed to the known value since a combined reconstruction of absorption and scattering is not possible from the continuous wave MC data set. The geometry of the slab was modeled by a finite element mesh of regular voxels of $2.5 \mathrm{~mm}$ size. The slab size was set to $(x, y$, $z)=(160 \mathrm{~mm}, 150 \mathrm{~mm}, 50 \mathrm{~mm})$. To obtain stable results we used the zero order Tikhonov regularization 

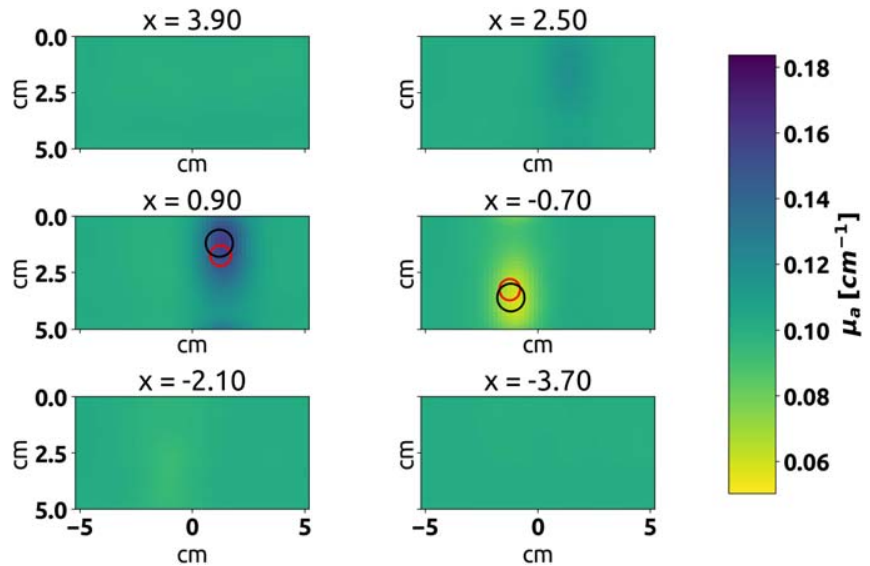

0.06

Figure 15. Same as figure 14 but showing slices where $X=$ constant.
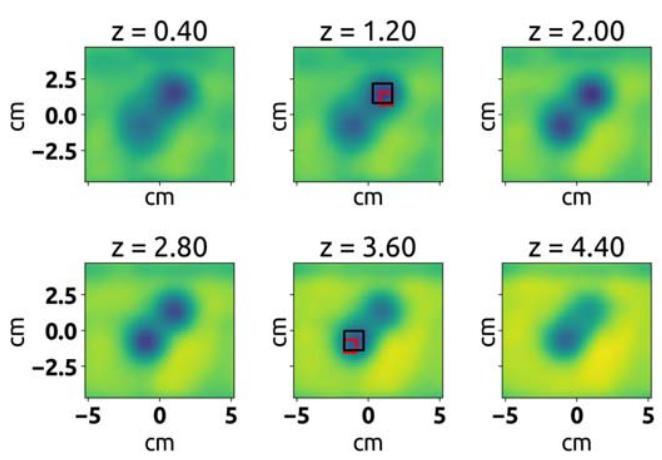

Figure 16. Selected slices of the reconstructed absorption coefficient 3D map at planes $Z=$ constant for the phantom experiment. At planes of maximum modulation for each inclusion, namely $z=1.2 \mathrm{~cm}$ for $I_{1}$ and $z=3.6 \mathrm{~cm}$ for $I_{2}$, the red squares represent the actual position and shape of the inclusions. The black squares correspond to the retrieved position and size.
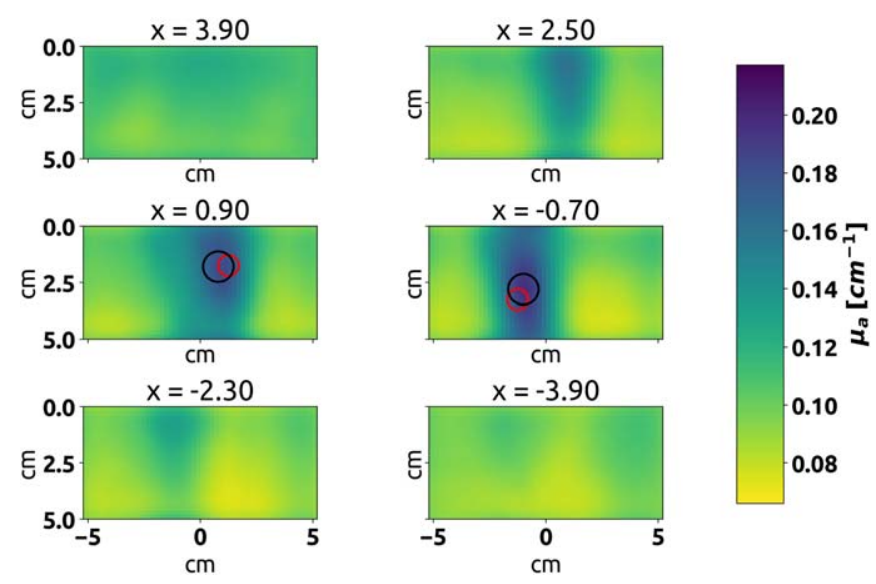

Figure 17. Same as figure 16 but showing slices where $X=$ constant.

which adds the term

$$
\tau \sum_{i}\left(\mu_{a, i}-\mu_{a, h o m}\right)^{2}
$$

to the minimization function. Here, $\mu_{a, h o m}$ refers to the absorption coefficient of the homogeneous medium. In this way, the reconstructed coefficients a, $\mathrm{i}$ (with i denoting the voxel number) are forced to stay close to the homogeneous absorption $\mu_{a \text {,hom }}=$ $0.1 \mathrm{~cm}^{-1}$ which was used as start value. The strength of the regularization is governed by the regularization parameter, $\tau$.

Figures 18 and 19 show a comparison of our tomosynthesis results (left column) with reconstructed slices for three different regularization parameters. With 


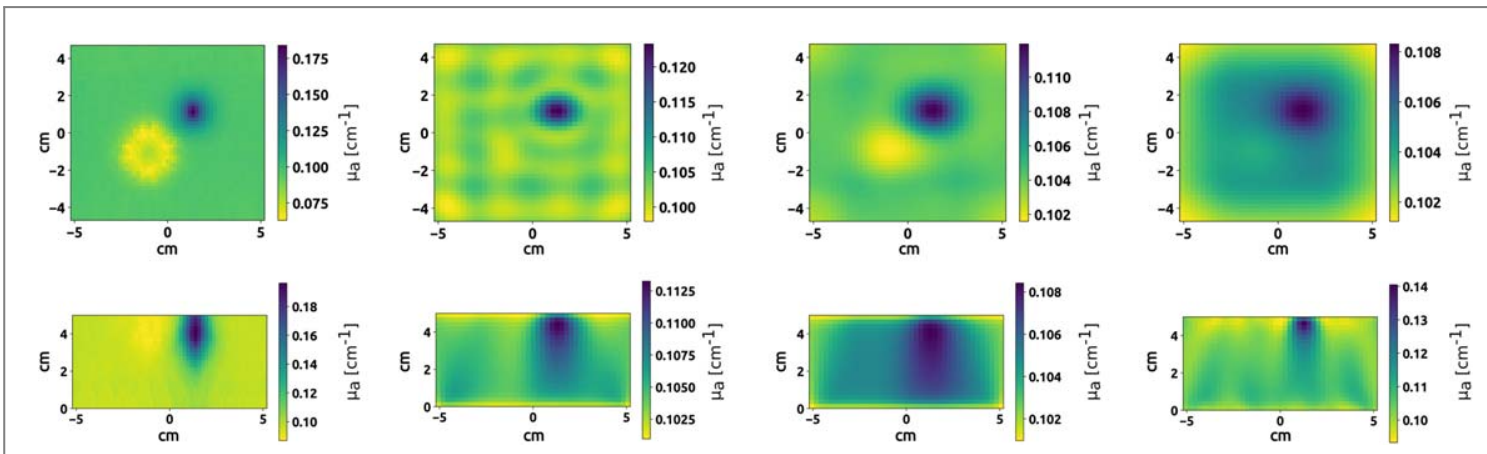

Figure 18. Comparison of reconstruction by tomosynthesis with $3 \mathrm{D}$ reconstruction by TOAST ++ using the MC data sets. The plots show the $x y$ plane and the $x z$ plane at the true locations of the inclusions for the Inclusion 1 with increased absorption. Left column: tomosynthesis intensity data based on percentiles (compare figures 7 and 8), Other columns: absorption obtained by TOAST ++ for $\tau=0.01$ ( 2 nd column), $\tau=0.1$ ( 3 rd column) and $\tau=1.0$ (last column).

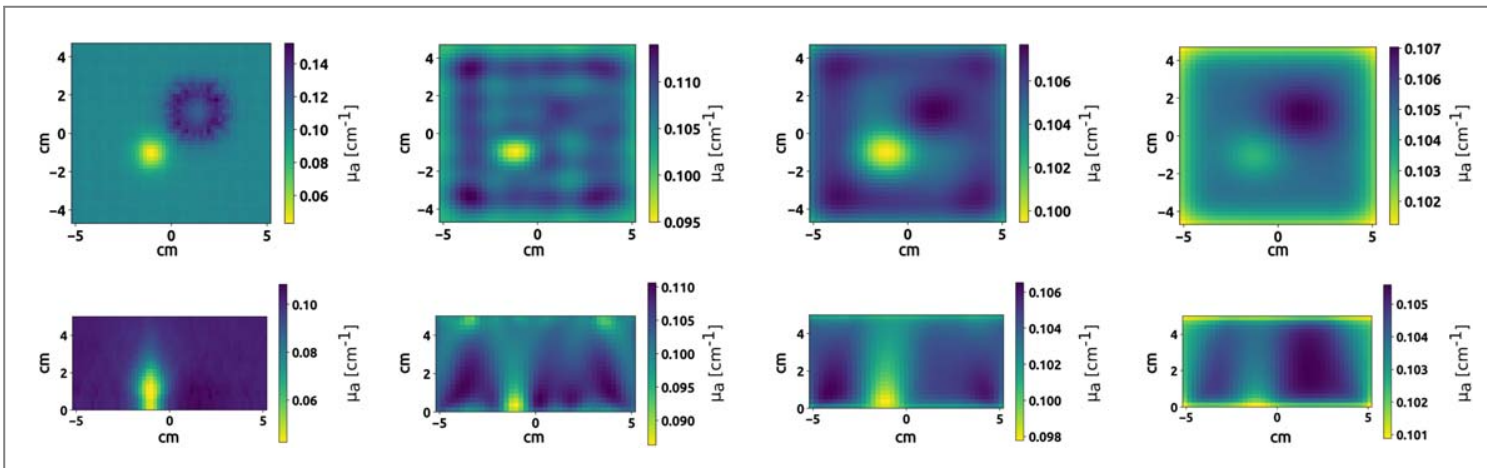

Figure 19. Comparison of reconstruction by tomosynthesis with $3 \mathrm{D}$ reconstruction by TOAST ++ using the MC data sets. The plots show the $x y$ plane and the $x z$ plane at the true locations of the inclusions for the Inclusion 2 with decreased absorption. Left column: tomosynthesis intensity data based on percentiles (compare figures 7 and 8 ), Other columns: absorption obtained by TOAST ++ for $\tau=0.01$ ( 2 nd column), $\tau=0.1$ (3rd column) and $\tau=1.0$ (last column).

the smallest regularization $(\tau=0.01,2$ nd column) the reconstructed slices show many artefacts. When the regularization parameter is increased to 0.1 (3rd column), both inclusions can be recognized in a similar manner to the tomosynthesis results. However, the $z$ positions are wrong. The inclusions appear strongly shifted to the edges of the phantom. Furthermore, the background has still several artefacts. By further increasing the regularization ( $\tau=1.0$, right column) the blurring along the $\mathrm{z}$ axis becomes very strong. Also, the second inclusion with the reduced absorption is difficult to recognize now. The absorption difference between inclusions and background recovered by TOAST ++ is less than 10 percent of the true difference. Moreover, the reconstructed background absorption is systematically overestimated. The true value is obtained only at the outer positions of the reconstruction grid not shown here. An improved result would require assumptions on the size and location of the inclusions in the reconstruction, similar to our assumptions in the absorption recovery by tomosynthesis. Overall, the intensity based tomosynthesis data as well as the related absorption results offer a superior localization compared to $3 \mathrm{D}$ reconstruction.

\subsubsection{Comparison with MRI guided DOT}

It is known that the strong underestimation of absorption differences in $3 \mathrm{D}$ reconstruction can be reduced by exploiting prior knowledge on object size and location. In a recent publication by Cochran et al (Cochran et al 2019) a $1.6 \mathrm{~cm}$ diameter object with an absorption to background contrast of 4:1 was successfully reconstructed from CW and time-resolved optical measurements using structural information from MR imaging of the phantom as a soft prior. The authors reconstructed a contrast of $2.7: 1$. With the tomosynthesis algorithm we obtained deviations between reconstructed and nominal absorption values in the same order. When using the size of the inclusion from the tomosynthesis reconstruction itself, absorption contrast ratios of $1.5: 1$ and $0.78: 1$ were reconstructed in the theoretical investigations for the nominal ratios of 2:1 and $0.5: 1$, respectively (compare with table 1 ). In case of the phantom experiment, we got a ratio of 2.2:1 for a nominal ratio 3.7:1 (table 2). When the true size of the inclusion was used as prior knowledge instead, ratios of 1.8:1 and $0.52: 1$ in the simulations and 3.1:1 in the experiment were obtained. These are fairly good results considering the simplicity and cost of our method. 
Table 2. Summary of the nominal and retrieved parameters, namely depth, diameter and absorption coefficient, for both inclusions in the phantom experiment. As stated for the MC simulations, the relative error in the retrieved absorption coefficients shows an stark reduction if some prior knowledge is used.

\begin{tabular}{|c|c|c|c|}
\hline \multicolumn{2}{|c|}{ Phantom experiments } & \multirow{2}{*}{$\begin{array}{c}\text { Inclusion } 1 \\
(1.25,1.10 \\
1.25) \mathrm{cm}\end{array}$} & \multirow{2}{*}{$\begin{array}{c}\text { Inclusion } 2 \\
(-1.25, \\
-1.10,3.75) \mathrm{cm}\end{array}$} \\
\hline Real inclusion & $\begin{array}{l}\text { Nominal } \\
\text { position }\end{array}$ & & \\
\hline & Diameter & $1 \mathrm{~cm}$ & $1 \mathrm{~cm}$ \\
\hline & $\langle t\rangle$ & $0.092 \mathrm{~ns}$ & $0.092 \mathrm{~ns}$ \\
\hline & Absorption & $0.26 \mathrm{~cm}^{-1}$ & $0.26 \mathrm{~cm}^{-1}$ \\
\hline \multirow[t]{6}{*}{$\begin{array}{c}\text { Tomosynthesis of } \\
\text { intensity data }\end{array}$} & $\begin{array}{l}\text { Retrieved } \\
\text { position }\end{array}$ & $\begin{array}{c}(0.82, \\
1.58 \\
1.2) \mathrm{cm}\end{array}$ & $\begin{array}{c}(-0.97 \\
-0.63,3.6) \mathrm{cm}\end{array}$ \\
\hline & Rel. error & $\begin{array}{c}(34 \%, \\
43 \%, 4 \%)\end{array}$ & $\begin{array}{c}(22 \%, \\
42 \%, 4 \%)\end{array}$ \\
\hline & FWHM & $1.5 \mathrm{~cm}$ & $1.5 \mathrm{~cm}$ \\
\hline & $\begin{array}{c}\text { Rel. } \\
\text { error } \\
\text { FWHM }\end{array}$ & $50 \%$ & $50 \%$ \\
\hline & $\langle t\rangle$ & $0.154 \mathrm{~ns}$ & $0.154 \mathrm{~ns}$ \\
\hline & $\begin{array}{c}\text { Rel. } \\
\text { error }\langle t\rangle\end{array}$ & $67 \% \mathrm{~ns}$ & $67 \%$ \\
\hline \multicolumn{2}{|c|}{ Absorption using estimated size } & $0.154 \mathrm{~cm}^{-1}$ & $0.154 \mathrm{~cm}^{-1}$ \\
\hline \multicolumn{2}{|l|}{ Relative deviation } & $-40 \%$ & $-40 \%$ \\
\hline \multicolumn{2}{|c|}{ Absorption using real size } & $0.217 \mathrm{~cm}^{-1}$ & $0.217 \mathrm{~cm}^{-1}$ \\
\hline \multicolumn{2}{|l|}{ Relative deviation } & $-16 \%$ & $-16 \%$ \\
\hline
\end{tabular}

\section{Conclusions}

We developed a simple and fast tomosynthesis method for obtaining the three-dimensional absorption map of a slab with breast-like optical properties. The approach is based on combining a scanner containing a cw point light source with a CCD camera to detect the transmitted intensity for each scan position at many lateral offsets. In this way, transmittance data are simultaneously detected for a large number of projection directions through the slab. Conventional tomosynthesis algorithms yield intensity data maps which contain only qualitative information on absorbers in the slab. We could overcome this limitation and generated absorption maps as well by using a perturbation model. For this analysis, the mean time of flight of photons through absorbers in the medium has to be known. We could estimate this time by using the conventional tomosynthesis intensity maps to derive the size of visible inclusions.

We successfully tested our method by MC simulations and by a phantom experiment. The large number of lateral offsets permitted us to compare tomosynthesis based on average data, on median data, and on selected percentiles. Investigations by means of the SSIM index showed that the median data are most convenient for the characterization of inclusions with unknown optical properties. The $80 \%$ percentile was advantageous for inclusions with increased absorption. The absorption difference with respect to the surrounding medium was always underestimated due to an overestimation of the size of the inclusion from the 3D intensity maps. When the true size of the inclusion was used as a prior, the underestimation could be reduced to about $10 \%$ in the experiments and to about $4 \%$ in the simulations.

The results of our reconstruction algorithm were compared with inverse calculations based on finite element, and they showed that tomosynthesis has the better localization performance for the utilized slab geometry. In addition, we compared our approach with results of a recent publication that performs DOT with prior geometrical knowledge obtained by MRI. We obtained fairly good results considering the simplicity and low cost of our method.

The obtained accuracy of absorption differences is a good basis for 3D resolved estimation of physiological parameters of tumours in the female breast. Corresponding investigations require the extension of the method by several cw sources with emission wavelengths preferably below $700 \mathrm{~nm}$, around $800 \mathrm{~nm}$, $980 \mathrm{~nm}$, and beyond $1000 \mathrm{~nm}$ to get access to deoxyand oxyhaemoglobin, to water and fat, and to collagen as the most important absorbers in breast tissue. Furthermore, the transmitted light has to be split with respect to these wavelengths to record separate images either by placing all subimages on the camera, or by application of several cheap cameras in parallel.

\section{Acknowledgments}

Authors like to thank Nvidia ${ }^{\boxplus}$ for donation of GeForce GTX Titan Xp GPU card as well as financial support from ANPCyT PICT $2018 \mathrm{~N}^{\circ} 1295$ and PICT Start Up $2018 \mathrm{~N}^{\circ} 4709$.

\section{ORCID iDs}

N A Carbone (1) https://orcid.org/0000-00025452-1165

D A Vera (1) https://orcid.org/0000-0002-6788-888X

\section{References}

Arridge S R 2011 Methods in diffuse optical imaging Philosophical Transactions of the Royal Society A: Mathematical, Physical and Engineering Sciences 369 4558-76

Brooksby B, Jiang S, Dehghani H, Pogue B W and Paulsen K D 2004 Magnetic resonance-guided near-infrared tomography of the breast Review of Scientific Instruments 75 5262-70

Brunet D, Vrscay E R and Wang Z 2011 On the mathematical properties of the structural similarity index IEEE Trans. Image Process. 21 1488-99

Carbone N A Github-tomosynthesisccd (https:/ / github.com/ nicocarbone/TomosynthesisCCD) Accessed: 2020-02-28

Carbone N A, Pomarico J A and Iriarte D I 2017 Gpu accelerated monte carlo simulation of light propagation in inhomogeneous fluorescent turbid media. application to whole field cw imaging Biomedical Physics and Engineering Express 3045012

Choe R et al 2005 Diffuse optical tomography of breast cancer during neoadjuvant chemotherapy: a case study with comparison to mri Med. Phys. 32 1128-39

Cochran J M, Busch D R, Lin L, Minkoff D L, Schweiger M, Arridge S and Yodha A G 2019 Hybrid time-domain and 
continuous wave diffuse optical tomography instrument with concurrent, clinical magnetic resonance imaging for breast cancer imaging J. Biomed. Opt. 24051409

Contini D, Martelli F and Zaccanti G 1997 Photon migration through a turbid slab described by a model based on diffusion approximation: I. theory Appl. Opt. 36 4587-99

Dierkes T, Grosenick D, Moesta K T, Möller M, Schlag P M, Rinneberg H and Arridge S 2005 Reconstruction of optical properties of phantom and breast lesion in vivo from paraxial scanning data Physics in Medicine \& Biology 502519

Dosselmann R and Yang X D 2011 A comprehensive assessment of the structural similarity index Signal, Image and Video Processing 5 81-91

Enfield L, Cantanhede G, Westbroek D, Douek M, Purushotham A, Hebden J and Gibson A 2011 Monitoring the response to primary medical therapy for breast cancer using threedimensional time-resolved optical mammography Technology in Cancer Research \& Treatment 10 533-47

Fang Q, Selb J, Carp S A, Boverman G, Miller E L, Brooks D H, Moore R H, Kopans D B and Boas D A 2011 Combined optical and $\mathrm{x}$-ray tomosynthesis breast imaging Radiology 258

Graves E E, Ripoll J, Weissleder R and Ntziachristos V 2003 A submillimeter resolution fluorescence molecular imaging system for small animal imaging Med. Phys. 30

Grosenick D, Wabnitz H, Moesta K T, Mucke J, Möller M, Stroszczynski C, Stößel J, Wassermann B, Schlag P M and Rinneberg H 2004 Concentration and oxygen saturation of haemoglobin of 50 breast tumours determined by time-domain optical mammography Physics in Medicine \& Biology 491165

Grosenick D, Hagen A, Steinkellner O, Poellinger A, Burock S, Schlag P, Rinneberg H and Macdonald R 2011 A multichannel time-domain scanning fluorescence mammograph: performance assessment and first in vivo results Rev. Sci. Instrum. 821024302

Grosenick D, Rinneberg H, Cubeddu R and Taroni P 2016 Review of optical breast imaging and spectroscopy J. Biomed. Opt. 21 091311

Haskell R C, Svaasand L O, Tsay T-T, Feng T-C, McAdams M S and Tromberg B 1994 Boundary conditions for the diffusion equation in radiative transfer J. Opt. Soc. Am. A 11

Li A et al 2003 Tomographic optical breast imaging guided by threedimensional mammography Appl. Opt. 42 5181-90

Mastanduno M A et al $2015 \mathrm{Mr}$-guided near-infrared spectra tomography increases diagnostic performance of breast mri Clinical Cancer Research 21 3906-12

Michaelsen K E, Krishnaswamy V, Shi L, Vedantham S, Karellas A, Pogue B W, Paulsen K D and Poplack S P 2016 Effects of breast density and compression on normal breast tissue hemodynamics through breast tomosynthesis guided nearinfrared spectral tomography J. Biomed. Opt. 21091316

Niklason L T et al 1997 Digital tomosynthesis in breast imaging Radiology 205 399-406

Pardini P A, Serra M W, Ranea-Sandoval H F, Pomarico J A and Iriarte D I 2015 Study of inks used in biomedical optics phantoms: stability and ageing J. Near Infrared Spectrosc. 23 219-25

Park J M, Franken E A Jr, Garg M, Fajardo L L and Niklason L T 2007 Breast tomosynthesis: present considerations and future applications Radiographics 27 S231-40
Poplack S P, Tosteson T D, Kogel C A and Nagy H M 2007 Digita breast tomosynthesis: initial experience in 98 women with abnormal digital screening mammography American Journal of Roentgenology 189 616-23

Rosebrock A How-to: Python compare two images (https:/ / pyimagesearch.com/2014/09/15/python-compare-twoimages $/$ \#::text=The\%20SSIM\%20method\%20is\% 20clearly,but\%20the\%20results\%20are\%20dramatic)

Sassaroli A, Martelli F and Fantini S 2006 Perturbation theory for the diffusion equation by use of the moments of the generalized temporal point-spread function: I. theory J. Opt. Soc. Am. 23

Sassaroli A, Pifferi A, Contini D, Torricelli A, Spinelli L, Wabnitx H, Ninni P D, Zaccanti G and Martelli F 2014 Forward solvers for photon migration in the presence of highly and totally absorbing objects embedded inside diffusive media J. Opt. Soc. Am. A 31

Scholkmann F, Kleiser S, Metz A J, Zimmermann R, Pavia J M, Wolf U and Wolf M 2014 A review on continuous wave functional near-infrared spectroscopy and imaging instrumentation and methodology Neuroimage 85 $6-27$

Schweiger M and Arridge S R 2014 The Toast++ software suite for forward and inverse modeling in optical tomography J. Biomed. Opt. 19 1-16

Scikit-image image development team. Structural similarity index (https://scikit-image.org/docs/dev/auto_examples/ transform/plot_ssim.html) Accessed: 2020-02-28

Taroni P, Paganoni A M, Ieva F, Pifferi A, Quarto G, Abbate F, Cassano E and Cubeddu R 2017 Non-invasive optical estimate of tissue composition to differentiate malignant from benign breast lesions: a pilot study Sci. Rep. 7 $1-11$

Van de Ven S M et al 2009 Diffuse optical tomography of the breast: preliminary findings of a new prototype and comparison with magnetic resonance imaging European radiology 19 1108

Vavadi H, Mostafa A, Zhou F, Uddin K S, Althobaiti M, Xu C, Bansal R, Ademuyiwa F, Poplack S and Zhu Q 2018 Compact ultrasound-guided diffuse optical tomography system for breast cancer imaging J. Biomed. Opt. 24021203

Wang L H, Jacques S L and Zheng L Q $1995 \mathrm{Mcml}$ monte carlo modeling of light transport in multilayered tissues Comput. Methods Programs Biomed 47 131-46

Wu T, Moore R H, Rafferty E A and Kopans D B 2004 A comparison of reconstruction algorithms for breast tomosynthesis Med. Phys. 31 2636-47

Xu C, Vavadi H, Merkulov A, Li H, Erfanzadeh M, Mostafa A, Gong Y, Salehi H, Tannenbaum S and Zhu Q 2016 Ultrasound-guided diffuse optical tomography for predicting and monitoring neoadjuvant chemotherapy of breast cancers: recent progress Ultrason. Imaging 38 5-18

Zhu Q, Chen N and Kurtzman S H 2003 Imaging tumor angiogenesis by use of combined near-infrared diffusive light and ultrasound Opt. Lett. $28337-9$

Zimmermann B B et al 2017 Multimodal breast cancer imaging using coregistered dynamic diffuse optical tomography and digital breast tomosynthesis J. Biomed. Opt. 22046008 